\title{
Die S3-Leitlinie Polytrauma
}

\section{Kernaussagen zu Thoraxtrauma, Schädel-Hirn-Trauma, Wirbelsäulenverletzungen und Extremitätenverletzungen}

\author{
Steffen Ruchholtz für die Autoren \\ Die vollständige Nennung der Autoren findet sich auf S. e52f.
}

\section{Übersicht}

Thoraxtrauma

Schädel-Hirn-Trauma

Wirbelsäulenverletzungen

\section{Thoraxtrauma}

\section{Diagnostik}

Grundlagen der Indikationsstellung zur Drainage/Dekompression des Pleuraraums sind die Untersuchung, die Wertung der erhobenen Befunde (Diagnose) und die Nutzen-Risiko-Abwägung (Diagnosesicherheit bei eingeschränkten diagnostischen Möglichkeiten, Zeitfaktor, Begleitumstände sowie die Risiken der Methode selbst).

Basisuntersuchung. Die körperliche Untersuchung des Patienten ist Voraussetzung für die Diagnosestellung und diese wiederum Voraussetzung für Therapiemaßnahmen. Nur durch die Untersuchung kann eine akut lebensbedrohliche Störung erkannt werden. Sie erscheint somit auch ohne wissenschaftlichen Beleg als zwingend erforderlich [89a].

Die Basisuntersuchung des Thorax in der Notfallsituation am Unfallort sollte im Check-up (nach Überprüfung und Sicherung der Vitalfunktionen) die Prüfung der Atemfrequenz und die Auskultation (Vorhandensein der Atemgeräusche, Seitengleichheit der Atemgeräusche) [14a, 36a, 39a, 40a] umfassen. Alle diese Zeichen sind mit signifikanten Pathologien korreliert oder haben unmittelbaren Einfluss auf medizinische Ent-
Schlüsselempfehlungen:

Untersuchung bei Thoraxtrauma

Eine klinische Untersuchung des Thorax und der Atemfunktion GoR A soll durchgeführt werden.

Die Untersuchung sollte mindestens die Bestimmung der GoR B Atemfrequenz und die Auskultation der Lunge umfassen. Eine wiederholte Untersuchung sollte erfolgen.

Die Inspektion (Seitendifferenz der Atemexkursion, Vorwölbung GoR 0 einer Seite, paradoxe Atmung), die Palpation (Schmerzen, Krepitationen, Hautemphysem, Instabilität) und die Perkussion (hypersonorer Klopfschall) des Thorax sowie die Pulsoxymetrie und, bei beatmeten Patienten, die Überwachung des Beatmungsdrucks können hilfreich sein.

scheidungen. Weitere sinnvolle Untersuchungen können die Inspektion (auf Verletzungszeichen, Symmetrie des Thorax, Symmetrie der Atemexkursion, paradoxe Atmung, Dyspnoe, Halsvenenfüllung) und die Palpation (Hautemphysem, Schmerzpunkte, Krepitationen, Instabilitäten des knöchernen Thorax) sein. Im Verlauf der weiteren Versorgung können die Überwachung des Beatmungsdrucks und die Pulsoxymetrie hinzukommen [55a]. 
Die verschiedenen Befunde sind zum Teil stark vom Untersucher, vom Patienten und von der Umgebung abhängig. So kann speziell Lärm die Auskultation erschweren oder unmöglich machen. Solche Umstände sind bei der Auswahl und Deutung der Primärdiagnostik zu beachten [36a,40a, 80a, 135a].

Umgebungsfaktoren wie Lärm sind bei der Auswahl und Deutung der Primärdiagnostik zu beachten.

Verlaufskontrolle. Die Prüfung der Atemfrequenz und die Auskultation sowie die Pulsoxymetrie und ggf. die Prüfung des Beatmungsdrucks sollten im Verlauf erfolgen, da sich eine Störung der Atemwege, eine Tubusfehllage, ein Spannungspneumothorax oder eine akute respiratorische Insuffizienz dynamisch entwickeln können. Die Verlaufsuntersuchung kann zudem als Erfolgskontrolle der eingeschlagenen Therapie dienen.

Schlüsselempfehlungen:

Diagnosestellung Pneumothorax

- Die Verdachtsdiagnose Pneumo- und/oder Hämatothorax soll bei einseitig abgeschwächtem oder fehlendem Atemgeräusch (nach Kontrolle der korrekten Tubuslage) gestellt werden. Das Fehlen eines solchen Auskultationsbefundes, insbesondere bei Normopnoe und thorakaler Schmerzfreiheit, schließt einen größeren Pneumothorax weitgehend aus.

- Die mögliche Progredienz eines kleinen, zunächst präklinisch nicht diagnostizierbaren Pneumothorax sollte in Betracht gezogen werden.
Im Verlauf kann sich eine Störung der Atemwege, eine Tubusfehllage, ein Spannungspneumothorax oder eine akute respiratorische Insuffizienz entwickeln. Um diese nicht zu übersehen, sollten mindestens die Bestimmung der Atemfrequenz und die Auskultation in regelmäßigen Abständen wiederholt werden.

Diagnosestellung Pneumothorax. Es sind aktuell keine Methoden zum sicheren präklinischen Nachweis oder Ausschluss eines Pneumothorax verfügbar. Dies ist klinisch nur durch Computertomografie (Ausschluss) möglich.

Die sichere Diagnose eines Pneumothorax (bzw. der sichere Ausschluss eines Pneumothorax) ist nur durch eine Computertomografie möglich.

Auskultation. In Tabelle 1 sind die Studien zur Diagnosegenauigkeit der Auskultation zusammengestellt. Die Spezifität eines einseitig abgeschwächten oder fehlenden Atemgeräuschs für das Vorliegen eines Hämato-/Pneumothorax ist mit 93-98\% sehr hoch. Der positive prädiktive Wert, das heißt die Wahrscheinlichkeit, mit der bei abgeschwächtem Atemgeräusch tatsächlich ein Pneumothorax vorliegt, ist mit 86-97\% ebenfalls sehr hoch [35a, 135a].

Voraussetzung ist die regelrechte Lage des endotrachealen Tubus (so vorhanden), die, soweit als möglich, vorher sichergestellt sein muss. Einschränkend muss konstatiert werden, dass die genannten Studien nicht

\section{Tabelle 1}

Diagnostische Wertigkeit eines pathologischen Auskultationsbefundes im Hinblick auf einen Hämato-/Pneumothorax.

\begin{tabular}{|c|c|c|c|c|}
\hline Studie & $\begin{array}{l}\text { Level } \\
\text { of Evidence }\end{array}$ & Patientenkollektiv & Sensitivität & Spezifität \\
\hline Hirshberg et al. 1988 [80a] & 1 & spitzes Trauma $(n=51)$ & $96 \%$ & $93 \%$ \\
\hline Wormland et al. 1989 [143a] & 3 & spitzes Trauma $(n=200)$ & $73,3 \%$ & $98,6 \%$ \\
\hline Thomson et al. 1990 [135a] & 1 & spitzes Trauma $(\mathrm{n}=102)$ & $96 \%$ & $94 \%$ \\
\hline Chen et al. 1997 [36a] & 3 & spitzes Trauma $(n=118)$ & $58 \%$ & $98 \%$ \\
\hline Chen et al. 1998 [35a] & 1 & überwiegend stumpfes Trauma $(n=148)$ & $84 \%$ & $97 \%$ \\
\hline Bokhari et al. 2002 [24a] & 2 & stumpfes Trauma $(n=523)$ & $100 \%$ & $99,8 \%$ \\
\hline Bokhari et al. 2002 [24a] & 2 & spitzes Trauma $(n=153)$ & $50 \%$ & $100 \%$ \\
\hline
\end{tabular}




\section{Tabelle 2}

Diagnostische Wertigkeit der Dyspnoe und Tachypnoe im Hinblick auf einen Hämato-/Pneumothorax.

\begin{tabular}{|lllll|}
\hline Studie & Level of Evidence & Patientenkollektiv & Sensitivität & Spezifität \\
\hline Wormland et al. 1989 [143a] & 3 & spitzes Trauma $(n=200)$ & $75,6 \%$ & $84,1 \%$ \\
\hline Hing et al. 2001 [79a] & 4 & spitzes Trauma $(n=153)$ & $72,7 \%$ & $95,5 \%$ \\
\hline Bokhari et al. 2002 [24a] & 2 & stumpfes Trauma $(n=523)$ & $42,8 \%$ & $99,6 \%$ \\
\hline Bokhari et al. 2002 [24a] & 2 & spitzes Trauma $(n=153)$ & $31,8 \%$ & $99,2 \%$ \\
\hline
\end{tabular}

\section{Tabelle 3}

Diagnostische Wertigkeit thorakaler Schmerzen im Hinblick auf einen Hämato-/Pneumothorax.

\begin{tabular}{|lllcc|}
\hline Studie & Level of Evidence & Patientenkollektiv & Sensitivität & Spezifität \\
\hline Bokhari et al. 2002 [24a] & 2 & stumpfes Trauma $(n=523)$ & $57,1 \%$ & $78,6 \%$ \\
\hline Bokhari et al. 2002 [24a] & 2 & spitzes Trauma $(n=153)$ & $25,0 \%$ & $91,5 \%$ \\
\hline
\end{tabular}

am Notfallort, sondern in der Notaufnahme im Krankenhaus durchgeführt wurden.

Dyspnoe. In mehreren Studien zeigte sich, dass die Normopnoe ein sehr sicheres Zeichen dafür ist, nach stumpfem Trauma das Vorliegen eines größeren Hämato-/Pneumothorax ausschließen zu können (hohe Spezifität). Dagegen bedeutet das Vorliegen einer Dyspnoe keineswegs im Umkehrschluss, dass ein Pneumothorax vorhanden ist (niedrige Sensitivität).

\section{Thorakaler Schmerz und Hämato-/Pneumothorax.}

Für den Stellenwert der Schmerzfreiheit liegt nur eine klinische Studie vor, die insbesondere für das spitze Trauma eine gute Spezifität aufzeigt [24a]. Wiederum ist dieser Befund nur in der Gesamtschau mit anderen Befunden von ausreichender diagnostischer Genauigkeit.

\section{Synopse thorakaler Schmerz, Dyspnoe, Auskultation.} Die Diagnosegenauigkeit für das Vorliegen eines Hämato-/Pneumothorax bei stumpfem Trauma in Abhängigkeit vom Vorliegen von thorakalen Schmerzen, Dyspnoe und eines einseitig abgeschwächten Atemgeräuschs bei der Auskultation ist in Tabelle $\mathbf{4}$ dargestellt.

Pneumothorax und Progredienz. Von Bedeutung ist die mögliche Progredienz eines anfänglich nicht symptomatischen Pneumothorax, insbesondere auch in der

\section{Tabelle 4}

Statistische Wahrscheinlichkeiten für das Vorliegen eines klinisch relevanten Hämatopneumothorax bei verschiedenen Befundkombinationen nach stumpfem Thoraxtrauma (Grundannahme: 10\% Prävalenz als Vortestwahrscheinlichkeit sowie Unabhängigkeit der Tests).

\begin{tabular}{llll|}
\hline $\begin{array}{l}\text { Thorakaler Schmerz } \\
\text { (Sensitivität 57\%, } \\
\text { Spezifität 79\%) }\end{array}$ & $\begin{array}{l}\text { Dyspnoe } \\
\text { (Sensitivität 43\%, } \\
\text { Spezifität 98\%) }\end{array}$ & $\begin{array}{l}\text { Auskultation } \\
\text { (Sensitivität 90\%, } \\
\text { Spezifität 98\%) }\end{array}$ & $\begin{array}{l}\text { Wahrscheinlich- } \\
\text { keit für Hämato-I } \\
\text { Pneumothorax }\end{array}$ \\
\hline+ & + & + & $>99 \%$ \\
\hline+ & + & - & $40 \%$ \\
\hline+ & - & + & $89 \%$ \\
\hline+ & - & - & $2 \%$ \\
\hline- & + & + & $98 \%$ \\
\hline- & + & - & $12 \%$ \\
\hline
\end{tabular}

Luftrettung. Die Progredienz von Pneumothoraces

kann individuell höchst unterschiedlich sein, und es ist das ganze Spektrum von einem stationären Befund bis zur rapiden Progredienz grundsätzlich möglich. Ge- 
wisse Hinweise lassen sich aus der Beobachtung kleiner Pneumothoraces ziehen. In einer prospektiv randomisierten Studie kam es bei 8 von 21 Patienten, bei denen ein okkulter Pneumothorax beobachtend behandelt worden war, zu einem progredienten Pneumothorax, in 3 Fällen zum Spannungspneumothorax. Alle diese Patienten waren beatmet [60a]. Die 3 Spannungspneumothoraces traten im Operationssaal, postoperativ nach Aufnahme auf der Intensivstation und während einer prolongierten Stabilisierungsphase auf, wobei genaue zeitliche Angaben in Stunden nach Trauma fehlen. Zumindest ist eine Zeitspanne von mindestens 30-60 min nach Klinikaufnahme anzunehmen. In einer weiteren prospektiv randomisierten Studie zur Therapie okkulter Pneumothoraces war die Progredienz des Pneumothorax in der Gruppe der konservativ behandelten Patienten (12,5\%) nicht größer als in der mittels Pleuradrainage therapierten (21\%) [25a]. Angaben über den Zeitraum der Pneumothoraxprogredienz wurden nicht gemacht.

Die meisten Experten sind der Meinung, dass die Progredienz eines Pneumothorax zu einem Spannungspneumothorax bei Patienten, die mit Überdruck beatmet werden, größer ist [13a], ohne dass dies quantifiziert werden kann.

Zusammenfassend legen die Daten nahe, dass kleine, klinisch nicht diagnostizierbare Pneumothoraces in der Regel relativ langsam progredient sind und somit keiner notfallmäßigen Dekompression im präklinischen Bereich bedürfen.

Kleine, klinisch nicht diagnostizierbare Pneumothoraces sind in der Regel wahrscheinlich nur langsam progredient und bedürfen somit keiner notfallmäßigen Dekompression.

\section{Diagnosestellung Spannungspneumothorax.}

Die überwiegende Mehrzahl der Experten sieht die Diagnose eines Spannungspneumothorax als gegeben,

\section{Schlüsselempfehlung:}

Diagnosestellung Spannungs-

\section{pneumothorax}

Die Verdachtsdiagnose Spannungspneumothorax sollte gestellt GoR B werden bei einseitig fehlendem Atemgeräusch bei der Auskultation der Lunge (nach Kontrolle der korrekten Tubuslage) und dem zusätzlichen Vorliegen von typischen Symptomen, insbesondere einer schweren respiratorischen Störung oder einer oberen Einflussstauung in Kombination mit einer arteriellen Hypotension. wenn lebensbedrohliche hämodynamische oder respiratorische Störungen vorliegen. Zyanose, Atemnot, Tachypnoe, eine Trachealdeviation zur Gegenseite und ein Abfall der Sauerstoffsättigung, aufgehobene Atemexkursion und vorgewölbter Hemithorax mit hypersonorem Klopfschall auf der erkrankten Seite sind mögliche respiratorische Zeichen. Zu den hämodynamischen Indikatoren können eine Stauung der Halsvenen, Tachykardie und schließlich Blutdruckabfall bis hin zum Kreislaufstillstand (pulslose elektrische Aktivität) kommen. Allerdings können viele dieser Zeichen oft nur bei genauer Untersuchung festgestellt werden und wurden bisher nicht systematisch untersucht. Daten von Traumapatienten liegen wenige vor; die meisten Informationen wurden aus der Beobachtung von Spannungspneumothoraces im intensiv-

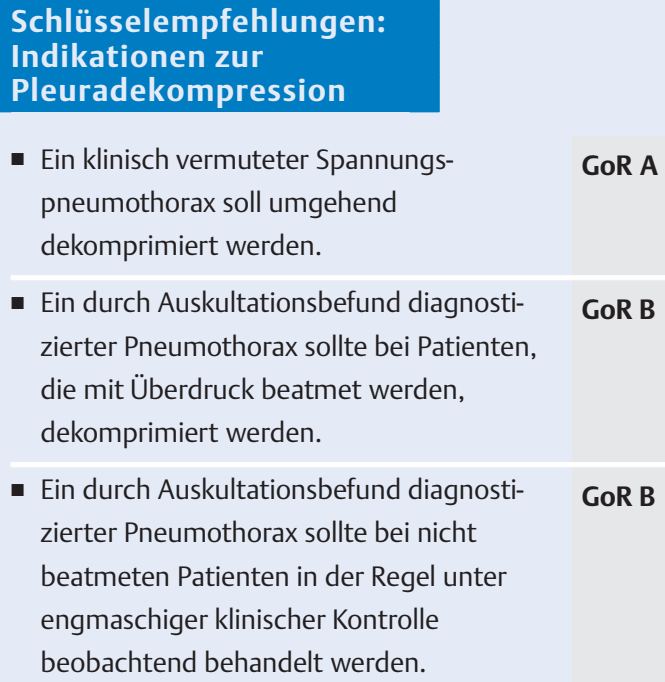

- Ein klinisch vermuteter SpannungsGoR A pneumothorax soll umgehend dekomprimiert werden.

- Ein durch Auskultationsbefund diagnostizierter Pneumothorax sollte bei Patienten, die mit Überdruck beatmet werden, dekomprimiert werden.

- Ein durch Auskultationsbefund diagnostizierter Pneumothorax sollte bei nicht beatmeten Patienten in der Regel unter engmaschiger klinischer Kontrolle beobachtend behandelt werden.

medizinischen Bereich gewonnen [90a].

Indikationen zur Pleuradekompression. Vergleichende Untersuchungen zwischen konservativer und intervenierender Therapie liegen nicht vor. Die Empfehlungen zum therapeutischen Vorgehen beruhen auf Expertenmeinung und Überlegungen zu Wahrscheinlichkeiten.

Spannungspneumothorax. Ein Spannungspneumothorax ist eine akut lebensbedrohliche Situation und führt unbehandelt in aller Regel zum Tod. Der Tod kann beim Auftreten von Zeichen der eingeschränkten Lungen- und Kreislauffunktion innerhalb von wenigen Minuten eintreten. Eine Alternative zur Dekompression gibt es nicht. Die Experten sind der Meinung, dass insbesondere bei eingetretener Kreislauf- oder Atemstörung eine sofortige notfallmäßige Entlastung durch- 
geführt werden sollte und der Zeitverlust durch den Transport - auch in ein in unmittelbarer Nähe gelegenes Krankenhaus - eine nicht zu vertretende Verzögerung darstellt.

Bei einem Spannungspneumothorax kann der Tod innerhalb von Minuten eintreten. Eine Alternative zur Dekompression gibt es nicht.

Diagnostizierter Pneumothorax. Ein großer Pneumothorax, der anzunehmen ist, wenn ein typischer Auskultationsbefund erhoben wird, stellt grundsätzlich eine Indikation zur Evakuierung des Pleuraraums dar. Wann dies geschehen muss - ob in der Präklinik oder erst im Krankenhaus -, ist im Einzelfall schwierig zu entscheiden, da das Risiko der Progredienz vom einfachen Pneumothorax zum Spannungspneumothorax sowie die Zeitdauer, die eine solche Entwicklung in Anspruch nehmen kann, variabel und schwer abzuschätzen sind. Hierzu existieren in der Literatur weder allgemeine Daten noch Risikofaktoren. Es gibt Hinweise, dass beim Vorliegen eines Thoraxtraumas bei intubierten Patienten häufiger mit einem Spannungspneumothorax bei Einlieferung im Krankenhaus zu rechnen ist als bei nicht intubierten Patienten. Insgesamt erscheint es den Experten jedoch plausibel, dass ein durch Auskultation diagnostizierter Pneumothorax bei einem beatmeten Patienten ein deutlich erhöhtes Risiko hat, sich zu einem Spannungspneumothorax zu entwickeln, und somit eine Indikation zur präklinischen Dekompression besteht.

Intubierte Patienten mit Thoraxtrauma haben möglicherweise ein größeres Risiko, auf dem Weg zum Krankenhaus einen Spannungspneumothorax zu erleiden, als nicht intubierte Patienten.

Ist ein Patient mit auskultatorisch diagnostiziertem Pneumothorax nicht beatmet, so erscheint das Risiko der Progredienz zum Spannungspneumothorax deutlich niedriger. Eine präklinische Dekompression erscheint hier nicht notwendig und eine beobachtende Therapie unter engmaschigem Monitoring und klinischer Kontrolle sollte erfolgen. Ist eine entsprechende Überwachung und klinische Kontrolle nicht gut möglich, zum Beispiel während Hubschraubertransporten, so besteht ein gewisses, nicht quantifizierbares Risiko, dass sich ein Spannungspneumothorax entwickelt und dass dieser nicht rechtzeitig bemerkt wird bzw. eine adäquate Therapie aus Platzgründen nicht möglich ist. In solchen Situationen kann beim Vorliegen entsprechender klinischer Zeichen und nach individueller Abwägung auch beim nicht intubierten Patienten eine Dekompression des Pneumothorax vor dem Transportbeginn erfolgen.

Thoraxtrauma ohne direkte Pneumothoraxdiagnose. Wenn kein Pneumothorax diagnostiziert wird (das heißt, wenn der Auskultationsbefund keine Seitendifferenz zeigt), so besteht auch grundsätzlich keine Indikation zu einer präklinischen Dekompression bzw. Evakuierung des Pleuraraums.

\section{Therapie}

Methoden. Ziel der Behandlung ist die Dekompression eines Überdrucks beim Spannungspneumothorax oder Spannungshämatothorax. In zweiter Linie kommt die Vermeidung einer Entwicklung vom einfachen Pneumothorax zum Spannungspneumothorax als Therapieziel in Betracht. Die permanente und möglichst komplette Evakuierung von Luft und Blut spielt beim präklinischen Notfall keine Rolle.

Ziel der Behandlung eines Spannungspneumothorax ist die Dekompression des Überdrucks. Bei einem einfachen Pneumothorax kann eine präklinische Entlastung in Erwägung gezogen werden, um eine Entwicklung zum Spannungspneumothorax zu vermeiden.

Therapie des Pneumothorax. Da es keine geeigneten vergleichenden Daten zu den 3 Methoden gibt, kann keine datenbasierte Empfehlung für eine Methode der Wahl ausgesprochen werden.

\section{Thoraxdrainage: Wirksamkeit und Komplikationen.}

Die Einlage einer Thoraxdrainage ist eine geeignete, hochwirksame, nicht komplikationsfreie Maßnahme zur Entlastung des Spannungspneumothorax, die insbesondere auch bei Versagen oder ungenügender Wirksamkeit der alternativen Maßnahmen zur Anwendung kommen muss. Sie stellt in der Regel auch die definitive Versorgung dar und hat die höchste Erfolgs-

Schlüsselempfehlungen:

Therapie des Pneumothorax

- Die Entlastung eines Spannungspneumothorax sollte durch eine GoR B Nadeldekompression, gefolgt von einer chirurgischen Eröffnung des Pleuraspalts mit oder ohne Thoraxdrainage, erfolgen.

- Ein Pneumothorax sollte - sofern die Indikation besteht - durch eine Thoraxdrainage behandelt werden. 
rate. In 79-95\% der Fälle war die präklinisch eingelegte Pleuradrainage die definitive und erfolgreiche Therapiemaßnahme [10a, 52a, 117a, 125a].

Umgekehrt weist die Pleuradrainage eine Versagensrate wegen Fehllagen oder ungenügender Wirksamkeit von 5,4-21\% (im Mittel 11,2\%) auf.

\section{Einfache chirurgische Eröffnung: Wirksamkeit und} Komplikationen. Die einfache chirurgische Eröffnung des Pleuraraums ist eine geeignete, wirksame und relativ einfache Maßnahme zur Entlastung eines Spannungspneumothorax. Sie ist allerdings nur für Patienten geeignet, die mit Überdruck beatmet werden, da nur bei ihnen immer ein positiver intrapleuraler Druck herrscht. Bei einem spontan atmenden Patienten entsteht ein negativer intrapleuraler Druck, durch den Luft durch die Thorakotomie in den Thorax eingesaugt werden kann.

Nadeldekompression: Wirksamkeit und Komplikationen. Die Nadeldekompression ist eine geeignete, häufig wirksame, einfache, aber nicht komplikationsfreie Maßnahme zur Drainage. Bei fehlender oder ungenügender Wirksamkeit ist unverzüglich eine chirurgische Dekompression bzw. die Einlage einer Drainage vorzunehmen.

Durchführung: Punktionsort. Als Punktionsort für die Einlage einer Pleuradrainage wird sowohl der 4.-6. Interkostalraum in der vorderen bis mittleren Axillarlinie [40a, 132a, 136a] als auch der 2.-3. Interkostalraum in der mittleren Klavikularlinie empfohlen. Als Orientierung kann beim Mann die Mamille dienen, kaudal derer in der Regel nicht punktiert werden soll, da durch eine zu tiefe Punktion die Gefahr einer abdominellen Fehllage und der Verletzung von abdominellen Organen steigt. $\mathrm{Zu}$ beachten ist, dass mit dem angegebenen Punktionsort der Durchtrittsort zwischen den Rippen gemeint ist. Die Hautinzision kann auch um einen Interkostalraum tiefer liegen (siehe Durchführung der Punktion).

Als Punktionsort bei Pneumothorax wird der 4. -6. Interkostalraum in der vorderen bis mittleren Axillarlinie oder der 2.-3. Interkostalraum in der mittleren Klavikularlinie empfohlen.

Für beide Punktionsorte sind deletäre Komplikationen in Form von Kasuistiken publiziert. In einer prospektiven Studie fand sich kein Einfluss der Punktionshöhe (2. -8. ICR) oder der lateralen Lage (MCL oder MAL) auf die Erfolgsrate bezüglich der Drainage von Pneumo- thoraces oder Hämatopneumothoraces nach spitzem Trauma [57a]. In einer Kohortenstudie wurden die Komplikationen bei Drainagenanlage im 2. -3. Interkostalraum in der mittleren Klavikularlinie $(\mathrm{n}=21)$ und im 4. -6. Interkostalraum in der vorderen Axillarlinie $(\mathrm{n}=80)$ analysiert [82a]. Zwar war die Rate der interlobären Fehllagen beim lateralen Zugang signifikant höher, allerdings war eine funktionelle Fehllage an beiden Punktionsorten vergleichbar häufig (6,3 vs. $4,5 \%)$.

Instrumentarium (Nadeldekompression). Die durchschnittliche Thoraxwanddicke betrug in einer Studie an Leichen ca. 3,2 cm mit einer hohen Streuung (Standardabweichung $1,5 \mathrm{~cm}$ ) [26a]. Britten bestätigte diese Ergebnisse durch sonografische Ausmessung und beobachtete, dass in 57\% der Fälle die Pleuratiefe über $3 \mathrm{~cm}$ und bei $4 \%$ der Probanden über $4,5 \mathrm{~cm}$ betrug. Er folgerte, dass eine mindestens $4,5 \mathrm{~cm}$ lange Nadel erforderlich sei, um bei der überwiegenden Mehrzahl der Fälle den Pleuraraum überhaupt erreichen zu können [27a]. Auch eine 4,5 cm lange Nadel kann zu kurz sein, um den Pleuraraum zu erreichen [28a].

Um bei der Mehrzahl der Patienten den Pleuraraum zu erreichen, ist eine mindestens $4,5 \mathrm{~cm}$ lange Punktionsnadel erforderlich. In Einzelfällen kann allerdings auch diese zu kurz sein.

Daten bezüglich des zu verwendenden Kanülendurchmessers oder der Art der Kanüle liegen keine vor. Um den Austritt von möglichst viel Luft zu ermöglichen, wird allgemein ein möglichst großer Kanülendurchmesser empfohlen.

Instrumentarium (Thoraxdrainage). Da es sich nach Trauma aber in mindestens 30\% der Fälle um kombinierte Pneumo-/Hämatothoraces handelt, wird befürchtet, dass zu dünne Drainagen zu schnell verstopfen können. Aus diesen Gründen wird bei Erwachsenen die Verwendung von 24-32-Fr-Drainagen empfohlen [16a, 83a, 132a, 136a].

Ableitungssysteme. Verlässliche Daten zur Frage, ob und wann eine Thoraxdrainage nach außen offen belassen werden kann oder nicht und welches Ableitungssystem ggf. angewendet werden soll, bestehen nicht. Eine einheitliche Expertenempfehlung kann ebenfalls nicht gegeben werden.

Aus theoretischen Überlegungen kann bei einem Patienten, der mit Überdruck beatmet wird, die Thoraxdrainage nach außen offen bleiben. 
Ist der Patient jedoch spontan atmend, besteht die Gefahr, dass bei Inspiration Luft von außen in den Pleuraspalt eingesogen werden kann und es dann zum Totalkollaps des Lungenflügels kommt. In dieser Situation ist die Anbringung eines Ventilmechanismus notwendig.

Heimlich-Ventil. Das Heimlich-Ventil stellt einen solchen kommerziell verfügbaren Ventilmechanismus dar. In experimentellen Untersuchungen wurde gezeigt, dass es bei 2 von 8 Ventilen zu einem Funktionsverlust des Ventils gekommen war, bei Überschreitung des Verfallsdatums war das Heimlich-Ventil sogar in 7 von 8 Fällen defekt [78a]. Neben einer Materialermüdung kann es auch durch koaguliertes Blut zum Funktionsverlust kommen. Diese Unsicherheit bezüglich der Funktionstüchtigkeit des Heimlich-Ventils bedingt ein unkalkulierbares Risikopotenzial, und bei seiner Anwendung ist eine engmaschige Überwachung notwendig.

Geschlossene Beutel- oder Kammersysteme. Durch das Anschließen eines geschlossenen Auffangbeutels kann zwar die Verschmutzungs- und Infektionsgefahr reduziert werden, bei einer entsprechend großen Luftfistel kann es jedoch schnell zu einer Füllung des Beutels mit Luft oder Blut und der erneuten Ausbildung eines Überdrucks mit Spannungssituation im Pleuraraum kommen.

Ein kommerziell verfügbares Ableitungssystem, bestehend aus einem Rücklaufventil, einem Beutel und einer Entlüftung, war in einer prospektiv randomisierten Studie bei Patienten nach Thorakotomie genauso erfolgreich wie ein Mehrkammersystem mit Wasserschloss. Verstopfungen wurden hier nicht beobachtet, obwohl die Drainagen postoperativ auch Blut bzw. blutiges Sekret förderten. Über den Einsatz im präklinischen Bereich bei traumatischen Hämatothoraces und bei Pneumothoraces liegen keine Erfahrungsberichte vor.

Die Verwendung eines einfachen Beutels ohne Ventil (zum Beipiel Kolostomiebeutel) [138a] kommt für Traumapatienten und Pneumothoraces nicht in Betracht.

Der sogenannte Xpand-Drain ist eine neue Entwicklung, bei der eine Sammelkammer über ein Ventil an die Pleuradrainage angeschlossen wird. An die Sammelkammer kann ein Sog appliziert werden und größere Flüssigkeitsmengen können über eine gesonderte Ableitung entleert werden.
Schlüsselempfehlung:

Eröffnung des Pleuraraums

Die Eröffnung des Pleuraraums sollte mittels Minithorakotomie erfolgen. Die Einlage der Thoraxdrainage sollte ohne Verwendung eines Trokars erfolgen.

Eröffnung des Pleuraraums. Die beste Technik wurde niemals mittels kontrollierter Studien untersucht. Die meisten Experten empfehlen eine standardisierte Technik: Die Anlage einer Pleuradrainage soll in steriler Technik durchgeführt werden. Nach der Hautdesinfektion wird beim nicht tief bewusstlosen Patienten eine Lokalanästhesie bis einschließlich der Pleura parietalis appliziert. Mit dem Skalpell erfolgt eine horizontale (quere) ca. 4-5 cm lange Hautinzision (bei Frauen aus kosmetischen Gründen auf entsprechender Höhe in der Submammärfalte) über der Rippe, die den zu punktierenden Interkostalraum unten begrenzt, oder eine Rippe tiefer. Es folgt die stumpfe Präparation der Subkutis und der Interkostalmuskulatur am Oberrand der Rippe mit einer stumpfen Schere oder Klemme. Die Pleura kann stumpf oder mittels eines kleinen Schnittes mit der Schere durchtrennt werden. Anschließend Einführen eines Fingers (steriler Handschuh) in den Pleuraspalt, um den korrekten Zugang zum Pleuraspalt zu verifizieren und sicherzustellen, dass keine Adhäsionen vorliegen, oder diese ggf. zu lösen [16a, 50a, 107a, 123a, 132a, 133a, 136a, 139a]. Soll nur die einfache Eröffnung des Brustkorbs erfolgen, so wird die Wunde mit einer sterilen Kompresse abgedeckt, die an einer Seite nicht verklebt wird (Ventilbildung).

Soll eine Thoraxdrainage eingelegt werden, so wird die Intervention fortgesetzt: Ein subkutaner Tunnel wird nicht von allen Experten als erforderlich angesehen [133a]. Ein Trokar zur blinden Präparation des Kanals sollte keinesfalls verwendet werden. Hierbei sind schwerwiegende Komplikationen aufgetreten wie etwa die Perforation des rechten Vorhofs bei einem Patienten mit Kyphoskoliose [104a] oder Perforationen der Lunge [65a]. Die Komplikationsraten in den Studien mit Trokartechnik liegen durchweg höher als in den Untersuchungen mit der chirurgischen Technik (11,0 vs. 1,6\%) (Anhang). In einer prospektiven Kohortenstudie (bei Intensivpatienten) zeigte sich, dass die Verwendung eines Trokars mit einer signifikant höheren Rate von Fehllagen einherging [120a]. Für den Moment der Durchtrennung der Pleura und des Einführens der Drainage wird von einigen Experten bei beatmeten Patienten empfohlen, eine kurze Beatmungspause zu machen, 
um die Gefahr einer Lungenparenchymverletzung bei geblähter Lunge zu reduzieren [65a,115a,116a].

Bei Verwendung der Trokartechnik treten mehr Komplikationen auf als bei Eröffnung des Pleuraraums mit der chirurgischen Technik.

Die Thoraxdrainage wird dann durch den präparierten Kanal eingelegt. Hierzu kann der parallel eingeführte Finger als Führungsschiene dienen. Die Drainagenspitze kann auch mit einer Klemme gefasst werden und mithilfe der steiferen Führungsmöglichkeit der Klemme geführt werden. Alternativ kann ein Trokar zur Führung der Drainage (nicht zur Präparation oder Perforation der Thoraxwand!) verwendet werden. Dazu ist sicherzustellen, dass die Spitze des Trokars keinesfalls über die Drainagenspitze heraussteht und keine Kraft beim Vorschieben der Drainage angewendet wird [136a].

Die Drainage ist durch Pflasterzügel oder eine Annaht gegen eine Dislokation zu sichern. Die Fixierung mittels einer selbstarretierenden Plastikschlinge ist auch möglich [105a].

Alternative Einführungstechniken. Eine Reihe von alternativen Techniken und Modifikationen der Minithorakotomie zur Entleerung des Pleuraspalts wurde publiziert. Die Beschreibung und kritische Wertung kann in der vollständigen Leitlinie nachgelesen werden.

\section{Schädel-Hirn-Trauma}

\section{Maßnahmen am Unfallort}

Vitalfunktionen. Aus ethischen Gründen sind prospektive randomisiert-kontrollierte Studien, die den Effekt einer Hypotonie und/oder Hypoxie auf das Behandlungsergebnis untersuchen, sicherlich nicht vertretbar. Es gibt aber viele retrospektive Studien [8b, 25], die ein deutlich schlechteres Behandlungsergebnis bei

\section{Schlüsselempfehlung:}

\section{Vitalfunktionen}

- Beim Erwachsenen sollte eine arterielle Normotension mit einem systolischen Blutdruck nicht unter $90 \mathrm{mmHg}$ angestrebt werden.

- Ein Absinken der arteriellen Sauerstoffsättigung unter $90 \%$ sollte vermieden werden.
Vorliegen einer Hypotonie oder Hypoxie belegen. Absolute Priorität der diagnostischen und therapeutischen Maßnahmen am Unfallort hat daher die Erkennung und nach Möglichkeit die sofortige Beseitigung aller Zustände, die mit einem Blutdruckabfall oder einer Abnahme der Sauerstoffsättigung im Blut einhergehen. Eine aggressive Therapie zur Anhebung des Blutdrucks und der Sauerstoffsättigung hat sich allerdings aufgrund von Nebenwirkungen nicht immer bewährt. Anzustreben sind eine Normoxie, Normokapnie und Normotonie.

Absolute Priorität der Maßnahmen am Unfallort hat die Erkennung und nach Möglichkeit sofortige Beseitigung aller Zustände, die mit einem Blutdruckabfall oder einer Abnahme der Sauerstoffsättigung im Blut einhergehen.

Bei insuffizienter Spontanatmung in jedem Fall, aber auch bei Bewusstlosen mit ausreichender Spontanatmung stellt sich die Frage nach der Intubation. Auch hierfür gibt es in der Literatur leider keine hochwertige Evidenz, die einen eindeutigen Nutzen der Maßnahme belegt. Hauptargument für die Intubation ist die effiziente Vermeidung einer Hypoxie. Diese droht bei Bewusstlosen auch bei suffizienter Spontanatmung, da es durch die gestörten Schutzreflexe zur Aspiration kommen kann. Hauptargument gegen die Intubation ist der hypoxische Schaden, der durch eine fehlerhafte Intubation eintreten kann. Bei der Entwicklung der als Vorlage dienenden DGNC-Leitlinie „Schädel-Hirn-Trauma im Erwachsenenalter“ [6b] bestand Einigkeit, dass der Nutzen überwiegt, und es wurde daher in dieser Leitlinie eine A-Empfehlung ausgesprochen. Dieser Konsens konnte für die aktuelle Polytrauma-Leitlinie nicht erzielt werden.

Neurologische Untersuchung. Für klinische Befunde findet sich in der Literatur eine prognostische Aussagekraft lediglich für das Vorliegen weiter, lichtstarrer Pupillen [8b,23b, 26b] und einer Verschlechterung des GCS-Wertes [8b, 17b, 23b], die beide mit einem schlechten Behandlungsergebnis korrelieren. Es gibt

Schlüsselempfehlung:

Neurologische Untersuchung

Die wiederholte Erfassung und DokuGoR A mentation von Bewusstseinsklarheit, Bewusstseinstrübung oder Bewusstlosigkeit mit Pupillenfunktion und Glasgow Coma Scale soll erfolgen. 
keine prospektiven randomisiert-kontrollierten Untersuchungen zur Steuerung der Therapie durch die klinischen Befunde. Da solche Studien sicherlich ethisch nicht vertretbar sind, wurde bei der Entwicklung der Leitlinie die Bedeutung der klinischen Untersuchung auf einen Empfehlungsgrad A heraufgestuft unter der derzeit nicht beweisbaren Annahme, dass ein möglichst frühzeitiges Entdecken lebensbedrohlicher Zustände mit entsprechenden therapeutischen Konsequenzen das Outcome verbessern kann.

Trotz verschiedener Schwierigkeiten [2b] hat sich die Glasgow Coma Scale (GCS) international als Einschätzung der momentan festzustellenden Schwere einer Hirnfunktionsstörung eingebürgert. Mit ihr können die Aspekte Augen öffnen, verbale Kommunikation und motorische Reaktion standardisiert bewertet werden. Die neurologischen Befunde, mit Uhrzeit in den Krankenunterlagen dokumentiert, sind entscheidend für den Ablauf der weiteren Behandlung. Kurzfristige Kontrollen des neurologischen Befundes zur Erkennung einer Verschlechterung sind unbedingt durchzuführen [8b, 10b].

Die neurologischen Befunde, mit Uhrzeit in den Krankenunterlagen dokumentiert, sind entscheidend für die weiteren Therapieentscheidungen. Der neurologische Befund sollte kurzfristig kontrolliert werden, um ggf. eine Verschlechterung zu erkennen.

Die alleinige Verwendung der GCS beinhaltet allerdings die Gefahr einer diagnostischen Lücke, insbesondere, wenn nur Summenwerte betrachtet werden. Dies gilt für das beginnende Mittelhirnsyndrom, das sich in spontanen Strecksynergismen bemerkbar machen kann, die nicht durch die GCS erfasst werden, und für Begleitverletzungen des Rückenmarks. Erfasst werden sollen daher die motorischen Funktionen der Extremitäten mit seitengetrennter Unterscheidung an Arm und Bein, ob keine, eine unvollständige oder eine vollständige Lähmung vorliegt. Hierbei sollte auf das Vorliegen von Beuge- oder Strecksynergismen geachtet werden. Sofern keine Willkürbewegungen möglich sind, muss an allen Extremitäten die Reaktion auf Schmerzreiz erfasst werden.

Zusätzlich zur GCS sollen die motorischen Funktionen der Extremitäten mit seitengetrennter Unterscheidung an Arm und Bein erfasst werden. Dabei ist insbesondere auf Lähmungen, Beuge- und Strecksynergismen sowie (wenn keine Willkürbewegungen möglich sind) an allen Extremitäten auf die Reaktion auf Schmerzreiz zu achten.
Liegt keine Bewusstlosigkeit vor, sind zusätzlich Orientierung, Hirnnervenfunktion, Koordination und Sprachfunktion zu erfassen.

Bei wachen Patienten sind zusätzlich Orientierung, Hirnnervenfunktion, Koordination und Sprachfunktion zu prüfen.

Hirnprotektive Therapie. Die Datenlage in der wissenschaftlichen Literatur hat bisher nicht den Nutzen weiter gehender, als spezifisch hirnprotektiv angesehener Therapieregimes belegen können. Derzeit kann keine Empfehlung für die prästationäre Gabe von 21-Aminosteroiden, Kalziumantagonisten, Glutamat-RezeptorAntagonisten oder Tris-(Tris[hydroxymethyl] aminomethan)-Puffer gegeben werden [8b, 11b, 18b, 29b].

\section{Schlüsselempfehlung: \\ Hirnprotektive Therapie}

Auf die Gabe von Glukokortikoiden soll verzichtet werden. GoR A

Therapie bei Verdacht auf stark erhöhten intrakraniellen Druck. In den Fällen mit Verdacht auf transtentorielle Herniation und den Zeichen des Mittelhirnsyndroms (Pupillenerweiterung, Strecksynergismen, Streckreaktion auf Schmerzreiz, progrediente Bewusstseinstrübung) kann die Hyperventilation als Behandlungsoption in der Frühphase nach Trauma eingesetzt werden [8b,25]. Richtwerte sind 20 Atemzüge/ min bei Erwachsenen. Die früher aufgrund ihrer oftmals eindrucksvollen hirndrucksenkenden Wirkung eingesetzte Hyperventilation hat allerdings aufgrund der induzierten Vasokonstriktion auch eine Reduktion der zerebralen Perfusion zur Folge. Dies beinhaltet das Risiko einer zerebralen Ischämie bei aggressiver Hyperventilation und damit der Verschlechterung des klinischen Outcomes [25].

\section{Schlüsselempfehlung: \\ Therapie bei Verdacht auf stark \\ erhöhten intrakraniellen Druck}

Bei Verdacht auf stark erhöhten intrakraniellen Druck, insbesondere

GoR 0 bei Zeichen der transtentoriellen Herniation (Pupillenerweiterung, Strecksynergismen, Streckreaktion auf Schmerzreiz, progrediente Bewusstseinstrübung), können die folgenden Maßnahmen angewandt werden:

- Hyperventilation

- Mannitol

- Hypertone Kochsalzlösung 
Die Gabe von Mannitol kann für einen kurzen Zeitraum (bis 1 Stunde) den intrakraniellen Druck (Intracranial Pressure [ICP]) senken [25]. Bei Verdacht auf transtentorielle Herniation kann die Gabe auch ohne Messung des ICP erfolgen.

Für die hirnprotektive Wirkung hypertoner Kochsalzlösungen gibt es bislang nur wenige Evidenzbelege. Im Vergleich zu Mannitol scheint die Mortalität etwas geringer zu sein. Diese Aussage beruht allerdings auf einer kleinen Fallzahl und ist statistisch nicht signifikant [28b].

Transport. Bei mehrfach Verletzten mit Symptomen eines begleitenden Schädel-Hirn-Traumas ist die Einweisung in eine Klinik mit adäquater Versorgungsmöglichkeit unumgänglich. Im Falle eines SchädelHirn-Traumas mit anhaltender Bewusstlosigkeit (GCS $\leq 8$ ), einer zunehmenden Eintrübung (Verschlechterung einzelner GCS-Werte), Pupillenstörung, Lähmung oder Anfällen sollte die Klinik auf jeden Fall über die Möglichkeit einer neurochirurgischen Versorgung intrakranieller Verletzungen verfügen [8b].

\section{Bei mehrfach Verletzten mit Verdacht auf Schädel-Hirn-Trauma: Einweisung in eine Klinik mit adäquater Versorgungsmöglichkeit.}

Zur Frage der Analgosedierung und Relaxierung für den Transport kann keine eindeutige Empfehlung ausgesprochen werden, da Studien fehlen, die eine positive Wirkung auf das Schädel-Hirn-Trauma belegen.

Bei perforierenden Verletzungen sollte der perforierende Gegenstand belassen werden, eventuell muss er abgetrennt werden. Verletzte intrakranielle Gefäße werden oft durch den Fremdkörper tamponiert, sodass das Herausziehen die Ausbildung einer intrakraniellen Blutung begünstigt. Die Entfernung muss daher unter operativen Bedingungen mit der Möglichkeit einer Blutstillung im verletzten Hirngewebe erfolgen. Auch wenn es keine prospektiven randomisiert-kontrollierten Studien zum optimalen Vorgehen bei perforierenden Verletzungen gibt, so ist aus pathophysiologischen Überlegungen dieses Vorgehen sinnvoll.

\section{Schlüsselempfehlung:}

Transport

- Bei perforierenden Verletzungen sollte der perforierende GegenGoR B stand belassen werden, eventuell muss er abgetrennt werden.

\section{Wirbelsäulenverletzungen}

\section{Diagnostik}

\author{
Schlüsselempfehlung: \\ Präklinische Diagnostik bei \\ Wirbelsäulenverletzungen \\ Eine gezielte körperliche Untersuchung \\ GoR A \\ inklusive der Wirbelsäule und der mit ihr \\ verbundenen Funktionen soll durchgeführt \\ werden.
}

Präklinische Diagnostik. Die Basisuntersuchung der Wirbelsäule in der Notfallsituation am Unfallort beinhaltet im Check-up (nach Überprüfung und Sicherung der Vitalfunktionen) beim ansprechbaren Patienten die orientierende neurologische Untersuchung der Sensibilität und Motorik. Ein segmentbezogenes neurologisches Defizit weist auf das Vorliegen einer Rückenmarksverletzung hin. Höhe und komplette/inkomplette Läsionen können eingrenzend bestimmt werden. Fehlende Rückenschmerzen sind noch kein sicheres Zeichen dafür, dass keine relevante Verletzung der Brust- oder Lendenwirbelsäule vorliegen kann [28c].
Die Basisuntersuchung der Wirbelsäule am Unfallort beinhaltet beim ansprechbaren Patienten die orientierende neurologische Untersuchung der Sensibilität und Motorik. Ein segmentbezogenes neurologisches Defizit weist auf das Vorliegen einer Rückenmarks- verletzung hin.

Die Inspektion (auf Verletzungszeichen, Verformungen) und das Abtasten (Druck-, Klopfschmerz, Stufen, Versetzungen, tastbare Lücken zwischen Dornfortsätzen) der Halswirbelsäule und des gesamten Rückens vervollständigen die Basisuntersuchung.

Die Bewertung des Unfallmechanismus kann Hinweise auf die Wahrscheinlichkeit einer Wirbelsäulenverletzung geben [20c].

Der Unfallmechanismus kann Hinweise auf die Wahrscheinlichkeit einer Wirbelsäulenverletzung geben. 
Schlüsselempfehlung:

Wirbelsäulenverletzung bei

bewusstlosen Patienten

Bei bewusstlosen Patienten soll bis zum

GoR A

Beweis des Gegenteils von dem Vorliegen

einer Wirbelsäulenverletzung ausgegangen werden.

Vorliegen einer Wirbelsäulenverletzung bei anderen Verletzungen. Die Koinzidenz von Wirbelsäulenverletzungen und bestimmten anderen Verletzungsmustern ist erhöht. Hierbei handelt es sich um rein statistische Wahrscheinlichkeiten.

Die Wahrscheinlichkeit einer Wirbelsäulenverletzung ist bei bestimmten anderen Verletzungsmustern erhöht.

Schlüsselempfehlung:

Diagnose einer instabilen

Wirbelsäulenverletzung

Beim Fehlen folgender 5 Kriterien ist

GoR A

davon auszugehen, dass keine instabile

Wirbelsäulenverletzung vorliegt:

- Bewusstseinsstörung

- neurologisches Defizit

- Wirbelsäulenschmerzen oder Muskelhartspann

- Intoxikation

- Extremitätentrauma

Instabile Wirbelsäulenverletzung. Um den präklinischen Patiententransport zu vereinfachen und um die radiologische Primärdiagnostik nach stumpfem Trauma der Wirbelsäule sinnvoll einzugrenzen, sind von mehreren Gruppen klinische Entscheidungsregeln erarbeitet worden.

Unter Beachtung der 5 Kriterien Bewusstseinsstörung, neurologisches Defizit, Wirbelsäulenschmerzen oder Muskelhartspann, Intoxikation und Extremitätentrauma wurden bei Domeier et al. lediglich 2 relevante Wirbelsäulenverletzungen übersehen [24c]. 13 stabile Wirbelsäulenläsionen, die keiner Osteosynthese bedurften, kamen hinzu, sodass sich eine Sensitivität von 95\% mit einem negativen Vorhersagewert von 99,5\% ergab. Die Studie bezog sich auf die gesamte Wirbelsäule und fand jeweils etwa 100 Frakturen der HWS, BWS und LWS.
Schlüsselempfehlung:

Schmerzen im Wirbelsäulenbereich

Akutschmerzen im Wirbelsäulenbereich nach Trauma sollten als

GoR B ein Hinweis auf eine Wirbelsäulenverletzung gewertet werden.

Wirbelsäulenverletzung ohne Rückenmarksbeteiligung. Die genannten Verletzungszeichen können sowohl bei einer knöchernen Wirbelsäulen- als auch bei einer umgebenden reinen Weichteilverletzung vorhanden sein. Es gibt keine präklinisch erhebbaren Befunde, die eine Wirbelsäulenverletzung mit Sicherheit beweisen oder ausschließen können. Äußere Verletzungszeichen - Verformungen, Druck-, Klopfschmerz, Stufen, Seitversatz, tastbare Lücken zwischen Dornfortsätzen - sind indirekte Hinweise auf das Vorliegen einer Verletzung der Wirbelsäule. Eine Beurteilung zur (Lagerungs-)Stabilität der Verletzung kann präklinisch nicht erstellt werden.

Es gibt keine präklinisch erhebbaren Befunde, die eine Wirbelsäulenverletzung mit Sicherheit beweisen oder ausschließen können. Äußere Verletzungszeichen sind indirekte Hinweise auf eine Verletzung der Wirbelsäule.

Maßgeblich für die Diagnose einer Rückenmarksschädigung ist das neurologische Defizit in der Sensibilität und/oder Motorik.

Die Höhe und komplette/inkomplette Läsionen sind nur eingrenzend bestimmbar. Eine Aussage über die Prognose der Verletzung ist somit am Unfallort abschließend nicht sicher möglich.

Eine unauffällige Neurologie schließt andererseits eine Wirbelsäulenverletzung mit Beteiligung des Rückenmarks nicht aus.

Ein unauffälliger (prä)klinischer neurologischer Untersuchungsbefund schließt eine Wirbelsäulenverletzung mit Beteiligung des Rückenmarks nicht aus.

\section{Versorgung}

Rettung. Bei der Rettung des Verletzten sind alle unphysiologischen Wirbelsäulenbewegungen, insbesondere Flexion, segmentale Rotation und Seitneigung, $\mathrm{zu}$ vermeiden. Es ist eine koordinierte Bewegung in 
Schlüsselempfehlungen:

Rettung bei Verdacht auf

Wirbelsäulenverletzung

- Bei akuter Lebensbedrohung (zum Beispiel Feuer/Explosionsgefahr), die nur durch sofortige Rettung aus dem Gefahrenbereich beseitigt werden kann, soll auch bei Verdacht auf eine Wirbelsäulenverletzung die sofortige und unmittelbare Rettung aus dem Gefahrenbereich erfolgen, ggf. auch unter Vernachlässigung von Vorsichtsmaßnahmen für den Verletzten.

- Die Halswirbelsäule soll vor der eigentlichen technischen Rettung immobilisiert werden.

die Ruheposition der Wirbelsäule, das heißt flache Rückenlage, mit genügend Helfern durchzuführen [6c]. Hilfsmittel wie die Schaufeltrage oder sogenannte Spineboards erleichtern die Rettung eines Wirbelsäulenverletzten in oben genannten Ruheposition aus ungünstiger Schadensortlage.

Bei der Bergung von Patienten mit Wirbelsäulenverletzung Wirbelsäulenbewegungen wie Flexion, segmentale Rotation und Seitneigung vermeiden.

Lagerung. Als erste präklinische Maßnahme für einen Unfallverletzten erfolgte bisher die Immobilisierung der HWS mit einer Zervikalstütze, auch wenn es hierzu keinen hohen Evidenzlevel gibt. Dabei erfolgt die Rücknahme der HWS in die Neutralposition. Kommt es dabei zu Schmerzen oder zur Zunahme eines neurologischen Defizits, ist eine Reposition in die Neutralstellung nicht durchzuführen.

Bei Vorliegen eines Schädel-Hirn-Traumas und Verdacht auf eine Halswirbelsäulenverletzung sollte abgewogen werden, ob eine starre Zervikalstütze angelegt wird oder eine anderweitige Ruhigstellung (zum Beispiel nur Vakuummatratze) erfolgen kann, um einen möglichen Anstieg des ICP zu verhindern [21c, 22c, 37c, 38c, 39c, 50c]. In einer anderen klinischen Studie konnte ein ICP-Anstieg bei korrekt angelegter starrer Zervikalstütze nicht nachgewiesen werden [39c]. Bei Anlegen einer starren Zervikalstütze bei SHT ist somit darauf zu achten, dass diese eine korrekte Größe hat und nicht zu fest zugezogen wird, um eine venöse Abflussstörung sicher auszuschließen. Zusätzlich ist hier eine Oberkörperhochlagerung anzustreben.
Die Ruhigstellung kann in der vorgegebenen Position auch auf der Vakuummatratze erfolgen. Diese erzielt die derzeit effektivste Immobilisation auch der gesamten Wirbelsäule.

Ein Verletztentragetuch auf der Vakuummatratze erleichtert die spätere klinische Umlagerung [8c]. Andere Hilfsmittel wie die Schaufeltrage oder Spineboards können die Wirbelsäule nur eingeschränkt immobilisieren.

\section{Schlüsselempfehlung: \\ Transport bei Wirbel- \\ säulenverletzung}

Der Transport sollte möglichst schonend GoR B und unter Schmerzfreiheit erfolgen.

Transport. Ein Wirbelsäulenverletzter sollte möglichst schonend - das heißt ohne weitere äußere Gewalteinwirkung zur Vermeidung von Schmerzen und evtl. Sekundärschäden - transportiert werden. Nach der Lagerung und Retention wird der Transport unter analgetischer Therapie durchgeführt. Den mechanisch schonendsten Transport ermöglicht ein Hubschrauber. Er bietet zudem unter Umständen Zeitvorteile beim notwendigen Transport eines Wirbelsäulenverletzten mit neurologischen Ausfällen in ein Zentrum.

Den mechanisch schonendsten (und meist schnellsten) Transport ermöglicht ein Hubschrauber.

\section{Therapie}

Vor- und Nachteile einer Kortisontherapie. Zusammenfassend hier die Vor- und Nachteile einer Gabe von Methylprednisolon entsprechend der aktuellen Literatur:

Gründe für eine Kortisontherapie:

1. Die NASCIS-II-Studie zeigte eine Verbesserung des motorischen Outcomes, sofern eine Methylprednisolontherapie innerhalb von 8 Stunden begonnen wurde [11c,12c]. Dieses Ergebnis war aber nur einseitig verifiziert und vom Untersucher abhängig.

2. Andere, methodisch schlechtere Studien haben ebenfalls einen Benefit einer Kortisontherapie gefunden, wobei hier überwiegend der Therapiebeginn erst bei Eintreffen in der Klinik evaluiert wurde. 
3. Die NASCIS-III-Studie zeigte einen größeren Effekt bei einer Therapiedauer von 48 Stunden, sofern der Therapiebeginn zwischen 3 und 8 Stunden nach Trauma lag [14c, 15c].

4. Relevante Nebenwirkungen wie Magenblutung sind nicht erhöht [27c], eine Häufung von Femurkopfnekrosen nach Hochdosiskortisontherapie konnte nicht nachgewiesen werden [64c].

5. Es gibt keine andere definitive pharmakologische Therapie für die Rückenmarksverletzung.

Gründe gegen eine Kortisontherapie:

1. In der NASCIS-I-Studie war kein relevanter Benefit nachweisbar [13c].

2. Der nachgewiesene Benefit war nur nachweisbar bei Patienten, die innerhalb von 8 Stunden therapiert wurden.

3. Der nachgewiesene Benefit war klein, von unsicherer klinischer Signifikanz und nach 1 Jahr noch kleiner als nach 6 Monaten [10c, 12c].

4. In der NASCIS-III-Studie gab es keine Placebokontrollgruppe [14c,15c].

5. Weitere Untersuchungen zeigten eine höhere Komplikationsrate bei den mit Kortison behandelten Patienten (Anstieg pulmonaler Komplikationen [29c, 31c], insbesondere bei älteren Patienten [42c], und gastrointestinale Blutungen [48c]).

6. Fehlender neurologischer Benefit in anderen Studien bei häufig unklarem Verletzungsmuster [30c, 42c, 48c, 49c].

Andere medikamentöse Therapien. Bei dem sogenannten neurogenen Schock unter Beachtung von eventuellen anderen verletzungsbedingten Blutungsquellen ist eine Infusionstherapie zur Kreislaufstabilisierung erforderlich. Die Menge der verabreichten Infusion bzw. der angestrebte arterielle Mitteldruck ist auch in der Expertenmeinung umstritten. Eine suffiziente analgetische Therapie ist notwendig.

Zielklinik. Die frühe operative Versorgung von Wirbelsäulenverletzungen mit Rückenmarksbeteiligung kann das neurologische Outcome verbessern [44c,52c].

Die frühe operative Versorgung (innerhalb von 72 Stunden) von HWS-Verletzungen mit neurologischen Ausfällen birgt kein erhöhtes Risiko für weitere, zusätzliche Komplikationen [44c].

Aus diesem Grund sollte insbesondere beim isolierten Wirbelsäulentrauma und bei nicht akuter Lebensbedrohung eine Versorgung im Wirbelsäulenzentrum angestrebt werden [60c]. Patienten mit einer Spinal-

\section{Schlüsselempfehlung:}

Patienten mit neurologischen Ausfällen und vermuteter

GoR B Wirbelsäulenverletzung sollten primär und mindestens in ein regionales Traumazentrum mit Wirbelsäulenchirurgie transportiert werden.

kanaleinengung, insbesondere zervikal, scheinen von einer frühen operativen Versorgung zu profitieren [4c]. Auch wenn nur eine geringe Evidenz besteht, so ist dennoch davon auszugehen, dass Patienten mit einer inkompletten Neurologie und partieller Verlegung des Spinalkanals von einer frühzeitigen Reposition und ggf. operativen Enttrümmerung profitieren könnten.

\section{Extremitätenverletzungen}

\section{Schlüsselempfehlungen: \\ Prioritäten bei Extremitätenverletzungen}

Stark blutende Verletzungen der Extremitäten, welche die

Vitalfunktion beeinträchtigen können, sollen mit Priorität versorgt werden.

Die Versorgung von Verletzungen der Extremitäten soll weitere Schäden vermeiden und die Gesamtrettungszeit beim Vorliegen weiterer bedrohlicher Verletzungen nicht verzögern.

Priorität. Die Sicherung der Vitalfunktionen sowie die Untersuchung von Kopf und Körperstamm sollten der Untersuchung der Extremitäten vorausgehen. Besonderheiten können sich bei Verletzungen der Extremitäten mit starkem Blutverlust ergeben [21d, 27d].

Starke und unmittelbar lebensbedrohliche Blutungen sollen sofort versorgt werden, auch unter Vernachlässigung des ABCDE-Schemas (siehe Schema des ATLS, Seite 133).

Die Feststellung von größeren externen, aber nicht unmittelbar lebensbedrohlichen Blutungen ist wichtig und erfolgt in der Regel unter „C“ (Circulation), während kleinere Blutungen im „Secondary Survey“ auffallen [21d]. 
Oberstes Gebot ist die Vermeidung weiterer Schäden, die Wiederherstellung und Aufrechterhaltung der Vitalfunktionen sowie der Transport in eine geeignete Klinik [11d,25d]!

Die Versorgung von Extremitätenverletzungen (Spülung/Wundversorgung/Schienung) sollte nicht die Rettungszeit beim Vorliegen weiterer lebensbedrohlicher Verletzungen verzögern [23d].

\section{Diagnostik}

Anamnese. Eine möglichst genaue Anamnese (Eigen-/ Fremdanamnese) zum Unfallhergang kann erhoben werden, um eine ausreichende Information zur einwirkenden Kraft und ggf. bei offenen Wunden zum Grad der Kontamination zu erhalten [2d,27d].

Neben der Unfallanamnese wie dem Zeitpunkt des Unfalles sollten, wenn möglich, Informationen (Allergien, Medikation, Vorerkrankungen sowie die Nüchternheit) eingeholt werden. In diesem Zusammenhang sollte auch eine Anamnese des Tetanusstatus erfolgen [21d,34d].

\section{Schlüsselempfehlung:}

Untersuchung der Extremitäten nach Unfall

Alle Extremitäten eines Verunfallten sollten präklinisch orientierend

GoR B untersucht werden. sichere und unsichere Frakturzeichen), Beurteilung der Durchblutung, Motorik und Sensibilität erfolgen. Auch sollte der Weichteilbefund evaluiert werden (geschlossene vs. offene Fraktur, Kompartment-Syndrom) [11d, 21d, 27d].

Lederbekleidung wie zum Beispiel Motorradbekleidung sollte, soweit möglich, belassen werden, da diese als Schienung mit Kompressionseffekt insbesondere für das Becken und die untere Extremität dient [14d,21d].

Die kapillare Reperfusion kann im Seitvergleich getestet werden [21d].

\section{Therapie}

Schlüsselempfehlung:

Allgemeines zur Therapie bei

Extremitätenverletzungen

Eine auch nur vermutlich verletzte Extremität $\quad$ GoR B sollte vor grober Bewegung/dem Transport des Patienten ruhiggestellt werden.

Allgemeines. Die Ruhigstellung einer verletzten Extremität ist eine wesentliche Maßnahme in der präklinischen Versorgung. Eine verletzte Extremität sollte vor grober Bewegung/dem Transport des Patienten ruhiggestellt werden. Gründe hierfür sind eine Schmerzlinderung, eine Verhinderung einer weiteren Weichteilschädigung/Blutung sowie die Verringerung des Risikos einer Fettembolie und eines neurologischen Schadens [21d,34d].

Untersuchung. Wache Patienten sollten zunächst nach Beschwerden und deren Lokalisation befragt werden. Bei Schmerzen kann eine frühzeitige und ausreichende Analgetikagabe erfolgen [21d]. Eine präklinische Untersuchung sollte durchgeführt werden [11d]. Die Untersuchung am Unfallort sollte in angebrachtem Maße zur Beurteilung der Schwere der Verletzung ohne wesentliche Verzögerung der Gesamtrettungszeit erfolgen [2d]. Die Untersuchung sollte orientierend vom Kopf zum Fuß erfolgen und nicht länger als 5 Minuten dauern [34d].

\footnotetext{
Die körperliche Untersuchung sollte orientierend vom Kopf zum Fuß erfolgen und nicht länger als 5 Minuten dauern.
}

Die Untersuchung sollte in der Folge Inspektion (Fehlstellung/Wunden/Schwellung/Durchblutung), Stabilitätsprüfung (Krepitation, abnorme Beweglichkeit,
Auch bei dem Verdacht auf eine Verletzung sollte eine Ruhigstellung erfolgen [10d,34d].

Bei Verletzung (oder Verdacht auf eine Verletzung) ist die Ruhigstellung der entsprechenden Extremität eine wesentliche Maßnahme der präklinischen Versorgung.

Hierzu sollten das proximal und distal der Verletzung gelegene Gelenk in die Immobilisation mit einbezogen werden [10d,11d,24d,34d]. Die verletzte Extremität sollte flach gelagert werden [4d]. Insbesondere bei verkürzten Femurfrakturen sollte eine Traktion/Ruhigstellung unter Traktion erfolgen, um die Blutung zu minimieren [2d, 21d]. Bei Ruhigstellung in einer abnormalen Position bieten sich Vakuumschienen an. Vakuumschienen sind rigide und können sich der Form der 
Extremität anpassen [21d]. Luftkammerschienen eignen sich zur Schienung von Verletzungen der oberen Extremität mit Ausnahme von schultergelenksnahen Verletzungen. An der unteren Extremität sind sie geeignet für die Ruhigstellung von Knie-, Unterschenkelund Fußverletzungen. Der Druck in den Luftkammerschienen bzw. die periphere Durchblutung muss nach Anlage regelmäßig überprüft werden [4d]. Vorteil der Luftkammerschiene ist ihr geringes Gewicht, Nachteil die Kompression der Weichteile, welche Sekundärschäden verursachen kann. Vakuumschienen sind deshalb zu favorisieren. Luftkammer- und Vakuumschienen sind für die Immobilisierung von schultergelenksnahen sowie Femurfrakturen ungeeignet [5d]. Eine Kühlung kann Schwellungen reduzieren und zur Schmerzlinderung führen [10d]. Oberschenkelverletzungen können ohne Komplikationen ausreichend mit einem Spineboard oder einer rigiden Schienung immobilisiert werden. Traktionssplinte müssen nicht unbedingt im Rettungsdienst mitgeführt werden.

Die proximal und distal der Verletzung liegenden Gelenke sollten in die Immobilisation mit einbezogen werden.

Traktionssplinte sollten insbesondere beim polytraumatisierten Patienten nicht verwendet werden, da es insbesondere bei diesen viele Kontraindikationen für den Gebrauch desselben gibt (Beckenfraktur/Knie-/Unterschenkel-/OSG-[Oberes Sprunggelenk-]Verletzung) [33d]. Aufgrund der bestehenden Kontraindikationen für den Gebrauch eines Traktionssplints, insbesondere beim Schwerstverletzten, werden diese nur selten angewandt. Dislozierte proximale Femurfrakturen sind ebenso Kontraindikationen für den Einsatz eines Traktionssplints [7d].

Traktionssplinte sollten insbesondere

beim polytraumatisierten Patienten

nicht verwendet werden.

Die Fotodokumentation von Wunden/offenen Frakturen kann erfolgen (Polaroid/digital). Die fotografische Dokumentation von Wunden, offenen Frakturen oder vorgefundenen Fehlstellungen erscheint sinnvoll, da sie gegebenenfalls die erneute Exposition präklinisch bereits verbundener Wunden oder ruhiggestellter Extremitäten in der Klinik verhindern kann, bis diese definitiv versorgt werden. Eine Fotodokumentation kann dem weiterbehandelnden Arzt bei der Bewertung der Verletzung helfen. Die Fotodokumentation darf die Versorgungs-/Rettungszeit nicht verlängern [2d,21d].
Schwere und Ausmaß der Verletzungen sind auf dem Notarztprotokoll zu dokumentieren und der Lokalbefund dem weiterbehandelnden Chirurgen nach Möglichkeit persönlich zu schildern [3d].

\section{Schlüsselempfehlung: Frakturen}

Grob dislozierte Frakturen und Luxationen sollten, wenn möglich, GoR B und insbesondere bei begleitender Ischämie der betroffenen Extremität/langer Rettungszeit annähernd präklinisch reponiert werden.

Reposition bei Frakturen. Vorrangiges Ziel ist die Sicherstellung der lokalen und peripheren Durchblutung. Eine anatomisch exakte Reposition ist nicht primäres Ziel. Wichtiger ist die achsgerechte und stabile Lagerung mit Herstellung einer adäquaten lokalen und peripheren Durchblutung [3d,5d]. Sollte keine Kompromittierung der neurovaskulären Versorgung der Extremität distal der Verletzung vorliegen, so kann prinzipiell auf eine Reposition verzichtet werden [2d]. Grob dislozierte Frakturen und Luxationen sollten, wenn möglich, und insbesondere bei begleitender Ischämie der betroffenen Extremität/langer Rettungszeit durch axialen Zug und manuelle Korrektur in die Neutralstellung oder in eine Stellung, die der Neutralstellung am nächsten kommt, präklinisch reponiert werden. Wichtig ist die Kontrolle der peripheren Durchblutung sowie der Motorik und Sensibilität (wo möglich) vor und nach der Reposition [3d-5d,11d,21d, 25d]. Ein zu starker Längszug ist zu vermeiden, da sich dadurch der Druck in den Muskellogen erhöht und sich die Durchblutung des Weichteilgewebes verschlechtert [3d, 5d].

Grob dislozierte Frakturen und Luxationen sollten, wenn möglich, durch axialen Zug und manuelle Korrektur in die Neutralstellung oder in eine Stellung, die der Neutralstellung am nächsten kommt, präklinisch reponiert werden.

Ein neurologisches oder vaskuläres Defizit distal der Fraktur erfordert einen sofortigen Repositionsversuch. Gleiches gilt bei Kompromittierung des Weichteilmantels/der Haut [21d]. Nach erfolgter Ruhigstellung sollte die erneute Kontrolle von Durchblutung, Sensibilität und peripherer Motorik erfolgen [2d,21d]. Sollte nach einem Repositionsversuch eine Verschlechterung der neurovaskulären Versorgung vorliegen, so ist die Extremität sofort wieder in die Ausgangsposition zu bringen und so bestmöglich zu stabilisieren [21d]. 
Liegt nach einem Repositionsversuch eine Verschlechterung der neurovaskulären Versorgung vor, ist die Extremität sofort wieder in die Ausgangsposition zu bringen und so bestmöglich zu stabilisieren.

Die Reposition von Sprunggelenksfrakturen/-luxationsfrakturen sollte nur durch den darin Erfahrenen erfolgen. Sonst ist eine Ruhigstellung in der vorgefundenen Position anzustreben [21d]. Bei den häufigen dislozierten Sprunggelenksfrakturen mit einer offensichtlichen Fehlstellung des Gelenks kann die Reposition noch am Unfallort erfolgen. Unter ausreichender Analgesie kann durch kontrollierten und kontinuierlichen Längszug mit beiden Händen an Kalkaneus und Fußrücken eine annähernd achsgerechte Stellung erreicht werden, welche dann entsprechend ruhiggestellt wird. Die erneute Dokumentation der Durchblutungsund neurologischen Situation sollte hiernach erfolgen.

Offensichtliche Frakturen der langen Röhrenknochen im Schaftbereich sind ebenfalls in dieser Weise zu behandeln. Gelenknahe Frakturen sind in ihrem Ausmaß schwer einzuschätzen und können nach Immobilisation in der schmerzarmen vorgefundenen Position ruhiggestellt und so schnell wie möglich der weiteren klinischen Diagnostik zugeführt werden [2d,34d].

Bei distalen Femurfrakturen sollte ein stärkerer Längszug vermieden werden, da dieser zur Kompromittierung der Poplitealgefäße führen kann. Eine leicht gebeugte Lagerung im Kniegelenk kann erfolgen (30-50 Grad) [4d].

\section{Schlüsselempfehlung:}

Offene Frakturen

Jede offene Fraktur sollte von groben Verschmutzungen

GoR B gereinigt und steril verbunden werden.
Schlüsselempfehlungen:

Blutungsstillende Maßnahmen

Aktive Blutungen sollten gemäß einem GoR B

Stufenschema behandelt werden:

- manuelle Kompression/Druckverband

- (Hochlagerung)

- Tourniquet

Indikationen für einen sofortigen Gebrauch

GoR 0 des Tourniquets/der Blutsperre können sein:

- lebensgefährliche Blutungen/Multiple Blutungsquellen an einer Extremität

- keine Erreichbarkeit der eigentlichen Verletzung

- mehrere Verletzte mit Blutungen

siert werden [26d,34d]. Am besten sind die Verbände erst wieder im OP zu öffnen [21d,26d].

Blutungsstillende Maßnahmen. Die blutungsstillenden Maßnahmen sollten einem Stufenschema folgen. Es sollte primär versucht werden, aktive Blutungen durch manuelle Kompression und Hochlagerung der Extremität zum Stillstand zu bringen. Anschließend sollte ein Druckverband angelegt werden. Ist dieser nicht ausreichend, sollte über dem ersten die Anlage eines zweiten Druckverbandes erfolgen. Als Hilfe zur fokussierten Kompression kann ein Verbandspäckchen verwendet werden. Bei weiterer Persistenz sollte versucht werden, eine Arterie proximal der Verletzung abzudrücken. Im Weiteren sollte, sofern möglich, ein Tourniquet angelegt werden. Ausnahmsweise kann ein Abklemmen des Gefäßes erfolgen (Amputation, längerer Transport, Halsgefäß, anatomische Lage machen Tourniquetgebrauch unmöglich) [3d, 4d, 11d, 21d, 32d].

In Regionen, in denen Tourniquets nicht appliziert werden können (proximale Extremitäten), können hämostatische Verbände eingesetzt werden [13d]. Eine Tourniquetanlage bedarf einer entsprechenden Analgesie [21d]. Am Oberarm kann eine Blutdruckmanschette mit $250 \mathrm{mmHg}$, am Oberschenkel mit $400 \mathrm{mmHg}$ angelegt werden [3d,5d]. Der Zeitpunkt der Tourniquetanlage sollte notiert werden [21d,22d,28d]. Das Tourniquet muss den arteriellen Blutfluss komplett unterbrechen. Ein fehlerhaft angelegtes Tourniquet kann die Blutung verstärken (nur Niederdrucksystem komprimiert) [22d]. Die Überprüfung der Effektivität sollte über ein Stoppen der Blutung, nicht über das Verschwinden des distalen Pulses erfolgen. Bei einer Fraktur kann es weiter aus dem Knochenmark bluten [22d]. 
Die Überprüfung der Effektivität blutstillender Maßnahmen erfolgt über ein Stoppen der Blutung.

Indikationen für einen sofortigen Gebrauch des Tourniquets können sein [22d]:

- extreme Blutungen/multiple Blutungsquellen an einer Extremität mit parallel notwendiger Sicherung der Vitalfunktion

- keine Erreichbarkeit der eigentlichen Verletzung (zum Beispiel eingeklemmte Person)

- Massenanfall von Verletzten

Die Anlage des Tourniquets sollte unter Berücksichtigung folgender Punkte erfolgen:

- Anlage so weit distal wie möglich, ca. $5 \mathrm{~cm}$ proximal der Verletzung

- Anlage direkt auf der Haut, um ein Abrutschen zu verhindern [22d,28d]

Bei Ineffektivität zunächst Versuch der Neuanlage mit mehr Kompression, erst danach Erwägung eines zweiten Tourniquets direkt proximal des ersten [22d]. Die Kühlung der mit einem Tourniquet versorgten Extremität kann bei langen Rettungszeiten die Ischämietoleranz erhöhen [15d].

Die Kühlung einer mit einem Tourniquet versorgten Extremität kann bei langen Rettungszeiten die Ischämietoleranz erhöhen.

Es gibt nur unzureichende Daten über die Dauer einer sicheren Anwendungszeit für Tourniquets. Die generelle Empfehlung liegt bei $2 \mathrm{~h}$, allerdings ist diese aufgrund von Daten entstanden, welche bei normovolämen Patienten mit pneumatischen Tourniquets gewonnen wurden [22d]. Sollte die Transportzeit bis zur operativen Versorgung weniger als $1 \mathrm{~h}$ betragen, so kann das Tourniquet belassen werden. Bei längeren Rettungszeiten ( $>1 \mathrm{~h}$ ) sollte bei einem stabilisierten Patienten versucht werden, das Tourniquet zu lösen. Sollte es zu einer erneuten Blutung kommen, so sollte das neu angelegte Tourniquet dann bis zur Versorgung im OP belassen werden [22d]. Das Tourniquet sollte nach 30 min auf seine weitere Notwendigkeit hin überprüft werden. Dieses ist nicht indiziert, wenn der Patient im Schock ist oder die Begleitumstände widrig (Personal) [12d].

Das Tourniquet sollte möglichst frühzeitig angelegt werden. Sollte ein Tourniquet nicht zum Verschwinden des distalen Pulses führen, so sollte ein zweiter direkt proximal des ersten platziert werden, um die Effektivität zu erhöhen. Es sollten keine Materialien unter dem
Tourniquet verwendet werden, da sie zur Lockerung desselben führen können. Tourniquets sollten direkt proximal der Wunde angelegt werden. Tourniquets sollten im Verlauf auf ihre Effektivität reevaluiert werden [17d]. Der Einsatz von Tourniquets ist mit einer höheren Überlebenswahrscheinlichkeit verbunden. Der Einsatz von Tourniquets vor Entstehen eines Schockes ist mit einer höheren Überlebenswahrscheinlichkeit verbunden, ebenso der schon präklinische Einsatz. Es trat keine dem Tourniqueteinsatz anzulastende Amputationsnotwendigkeit auf.

\section{Schlüsselempfehlung: Amputationen}

Das Amputat sollte grob gereinigt und in sterile, feuchte GoR B Kompressen gewickelt werden. Es sollte indirekt gekühlt transportiert werden.

Amputationen. Neben einer Blutstillung sollte der Amputationsstumpf geschient und steril verbunden werden. Nur grobe Verschmutzungen sollten entfernt werden [3d,4d]. Das Amputat ist zu asservieren. Knochenteile oder amputierte Gliedmaßen sind nach Möglichkeit vom Unfallort mitzunehmen oder ggf. nachbringen zu lassen.

Das Amputat in sterile, feuchte Kompressen einwickeln und gekühlt, wenn möglich mit der „Doppelbeutelmethode“ verpackt, transportieren. Dabei wird das Amputat in einen inneren Plastikbeutel mit sterilen, feuchten Kompressen verpackt. Dieser Beutel wird in einen Beutel mit Eiswasser ( $1 / 3$ Eiswürfel, 2/3 Wasser) gelegt und verschlossen. Dabei ist ein sekundärer Kälteschaden zu vermeiden (kein direkter Kontakt von Eis oder Cool Pack mit dem Gewebe) [2d,3d,4d, 19d].

Amputationen beeinflussen die Auswahl der Zielklinik und sind entsprechend anzukündigen [2d,3d].

Amputationen beeinflussen die Auswahl der Zielklinik und sind in dieser entsprechend anzukündigen. 
Kernaussagen

Thoraxtrauma

Eine klinische Untersuchung des Thorax und der Atemfunktion soll durchgeführt werden.

Die Untersuchung sollte mindestens die Bestimmung der Atemfrequenz und die Auskultation der Lunge umfassen. Eine wiederholte Untersuchung sollte erfolgen.

Die Inspektion (Seitendifferenz der Atemexkursion, Vorwölbung einer Seite, paradoxe Atmung), die Palpation (Schmerzen, Krepitationen, Hautemphysem, Instabilität) und die Perkussion (hypersonorer Klopfschall) des Thorax sowie die Pulsoxymetrie und, bei beatmeten Patienten, die Überwachung des Beatmungsdrucks können hilfreich sein.

Die Verdachtsdiagnose Pneumo- und/oder Hämatothorax soll bei einseitig abgeschwächtem oder fehlendem Atemgeräusch (nach Kontrolle der korrekten Tubuslage) gestellt werden. Das Fehlen eines solchen Auskultationsbefundes, insbesondere bei Normopnoe und thorakaler Schmerzfreiheit, schließt einen größeren Pneumothorax weitgehend aus.

Die mögliche Progredienz eines kleinen, zunächst präklinisch nicht diagnostizierbaren Pneumothorax sollte in Betracht gezogen werden.

Die Verdachtsdiagnose Spannungspneumothorax sollte gestellt werden bei einseitig fehlendem Atemgeräusch bei der Auskultation der Lunge (nach Kontrolle der korrekten Tubuslage) und dem zusätzlichen Vorliegen von typischen Symptomen, insbesondere einer schweren respiratorischen Störung oder einer oberen Einflussstauung in Kombination mit einer arteriellen Hypotension.

Ein klinisch vermuteter Spannungspneumothorax soll umgehend dekomprimiert werden.

Ein durch Auskultationsbefund diagnostizierter Pneumothorax sollte bei Patienten, die mit Überdruck beatmet werden, dekomprimiert werden.

Ein durch Auskultationsbefund diagnostizierter Pneumothorax sollte bei nicht beatmeten Patienten GoR B in der Regel unter engmaschiger klinischer Kontrolle beobachtend behandelt werden.

Die Entlastung eines Spannungspneumothorax sollte durch eine Nadeldekompression, gefolgt von einer chirurgischen Eröffnung des Pleuraspalts mit oder ohne Thoraxdrainage, erfolgen.

Ein Pneumothorax sollte - sofern die Indikation besteht - durch eine Thoraxdrainage behandelt werden.

Die Eröffnung des Pleuraraums sollte mittels Minithorakotomie erfolgen. Die Einlage der Thoraxdrainage sollte ohne Verwendung eines Trokars erfolgen.

\section{Schädel-Hirn-Trauma}

Beim Erwachsenen sollte eine arterielle Normotension mit einem systolischen Blutdruck nicht unter $90 \mathrm{mmHg}$ angestrebt werden.

Ein Absinken der arteriellen Sauerstoffsättigung unter $90 \%$ sollte vermieden werden.

Die wiederholte Erfassung und Dokumentation von Bewusstseinsklarheit, Bewusstseinstrübung oder

Bewusstlosigkeit mit Pupillenfunktion und Glasgow Coma Scale soll erfolgen.

Auf die Gabe von Glukokortikoiden soll verzichtet werden.

Bei Verdacht auf stark erhöhten intrakraniellen Druck, insbesondere bei Zeichen der transtentoriellen Herniation (Pupillenerweiterung, Strecksynergismen, Streckreaktion auf Schmerzreiz, progrediente Bewusstseinstrübung), können die folgenden Maßnahmen angewandt werden:

- Hyperventilation

- Mannitol

- hypertone Kochsalzlösung

Bei perforierenden Verletzungen sollte der perforierende Gegenstand belassen werden, eventuell muss er abgetrennt werden. 


\section{Kernaussagen}

\section{Wirbelsäulenverletzungen}

Eine gezielte körperliche Untersuchung inklusive der Wirbelsäule und der mit ihr verbundenen Funktionen soll durchgeführt werden.

Bei bewusstlosen Patienten soll bis zum Beweis des Gegenteils von dem Vorliegen einer

GoR A Wirbelsäulenverletzung ausgegangen werden.

Beim Fehlen folgender 5 Kriterien ist davon auszugehen, dass keine instabile Wirbelsäulenverletzung

GoR A vorliegt:

- Bewusstseinsstörung

- neurologisches Defizit

- Wirbelsäulenschmerzen oder Muskelhartspann

- Intoxikation

- Extremitätentrauma

Akutschmerzen im Wirbelsäulenbereich nach Trauma sollten als ein Hinweis auf eine Wirbelsäulenverletzung

GoR B gewertet werden.

Bei akuter Lebensbedrohung (zum Beispiel Feuer/Explosionsgefahr), die nur durch sofortige Rettung aus dem Gefahrenbereich beseitigt werden kann, soll auch bei Verdacht auf eine Wirbelsäulenverletzung die sofortige und unmittelbare Rettung aus dem Gefahrenbereich erfolgen, ggf. auch unter Vernachlässigung von Vorsichtsmaßnahmen für den Verletzten.

Die Halswirbelsäule soll vor der eigentlichen technischen Rettung immobilisiert werden.

GoR A

Der Transport sollte möglichst schonend und unter Schmerzfreiheit erfolgen.

GoR B

Patienten mit neurologischen Ausfällen und vermuteter Wirbelsäulenverletzung sollten primär und

GoR B mindestens in ein regionales Traumazentrum mit Wirbelsäulenchirurgie transportiert werden.

\section{Extremitätenverletzungen}

Stark blutende Verletzungen der Extremitäten, welche die Vitalfunktion beeinträchtigen können, sollen mit Priorität versorgt werden.

Die Versorgung von Verletzungen der Extremitäten soll weitere Schäden vermeiden und die Gesamtrettungszeit beim Vorliegen weiterer bedrohlicher Verletzungen nicht verzögern.

Alle Extremitäten eines Verunfallten sollten präklinisch orientierend untersucht werden.

Eine auch nur vermutlich verletzte Extremität sollte vor grober Bewegung/dem Transport des Patienten ruhiggestellt werden.

Grob dislozierte Frakturen und Luxationen sollten, wenn möglich, und insbesondere bei begleitender Ischämie der betroffenen Extremität/langer Rettungszeit annähernd präklinisch reponiert werden. Jede offene Fraktur sollte von groben Verschmutzungen gereinigt und steril verbunden werden.

Aktive Blutungen sollten gemäß einem Stufenschema behandelt werden:

- manuelle Kompression/Druckverband

- (Hochlagerung)

- Tourniquet

Indikationen für einen sofortigen Gebrauch des Tourniquets/der Blutsperre können sein:

- lebensgefährliche Blutungen/Multiple Blutungsquellen an einer Extremität

- keine Erreichbarkeit der eigentlichen Verletzung

- mehrere Verletzte mit Blutungen

Das Amputat sollte grob gereinigt und in sterile, feuchte Kompressen gewickelt werden. Es sollte indirekt gekühlt transportiert werden. 


\section{Über die Autoren}

\section{Steffen Ruchholtz}

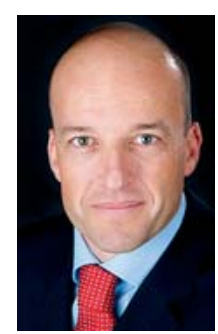

Jahrgang 1963, studierte in Ulm und München Medizin und ist Direktor der Klinik für Unfall-, Hand- und Wiederherstellungschirurgie am Universitätsklinikum Gießen-Marburg; Standort Marburg. Nach der Habilitation an der Universität Duisburg-Essen wechselte er 2007 auf den Lehrstuhl für Unfallchirurgie der Universität Marburg. Prof. Ruchholtz ist Sprecher des Arbeitskreises zur Umsetzung Weißbuch/Traumanetzwerk (AKUT) der DGU sowie Mitglied des Präsidiums der Deutschen Gesellschaft für Unfallchirurgie.

\section{Korrespondenzadresse}

Prof. Dr. Steffen Ruchholtz

Klinik für Unfall-, Hand- und Wiederherstellungschirurgie Universitätsklinikum Gießen-Marburg; Standort Marburg

Baldingerstraße

35043 Marburg

Telefon: $06421 / 28-66216$

Fax: 06421/28-66721

E-Mail: ruchholt@med.uni-marburg.de

PD Dr. med. Hermann Bail Charité - Campus Virchow Klinikum Klinik für Unfall- und Wiederherstellungs-chirurgie Augustenburger Platz 1

13353 Berlin

Dr. med. Mark Bardenheuer Universitätsklinikum Mannheim Theodor-Kutzer-Ufer 1-3

68167 Mannheim

Dr. med. Michael Bayeff-Filloff

Klinikum Rosenheim

Zentrale Notaufnahme

Pettenkoferstr. 10

83022 Rosenheim

Prof. Dr. med. Alexander Beck

Juliusspital Würzburg

Abteilung für Orthopädie, Unfall- und Wiederherstellungschirurgie

Juliuspromenade 19

97070 Würzburg
PD Dr. med. Achim Biewener Universitätsklinikum Dresden Klinik für Unfall- und Wiederherstellungschirurgie Fetscherstr. 74

01307 Dresden

Prof. Dr. med. Bertil Bouillon Kliniken der Stadt Köln gGmbH

Krankenhaus Merheim

Klinik für Unfallchirurgie, Orthopädie und Sporttraumatologie 51058 Köln

Dr. med. Marc Fischbacher Universitätsklinikum Essen Klinik für Unfallchirurgie Hufelandstr. 55

45147 Essen

Dr. med. Sebastian Hentsch Unfallkrankenhaus Berlin Klinik für Unfallchirurgie und Orthopädie Warener Str. 7

12683 Berlin

Dr. med. Ewald Hüls

Allgemeines Krankenhaus Celle Klinik für Unfallchirurgie, Orthopädie und Neurotraumatologie Siemensplatz 4 29223 Celle

PD Dr. med. Karl-Georg Kanz Klinikum der Universität München Chirurgische Klinik und Poliklinik Nussbaumstr. 20 80336 München

Prof. Dr. med. Christian K. Lackner Klinikum der Universität München Institut für Notfallmedizin und Medizinmanagement Schillerstr. 53

80336 München

Dr. med. Tobias Lindner

Charité - Campus Virchow Klinikum

Klinik für Unfall- und Wiederherstellungschirurgie

Augustenburger Platz 1

13353 Berlin

Dipl.-Med. Ivan Marintschev

Universitätsklinikum Jena

Klinik für Unfall-, Hand- und Wiederherstellungschirurgie Erlanger Allee 101

07747 Jena 
PD Dr. med. Gerrit Matthes

Unfallkrankenhaus Berlin

Klinik für Unfallchirurgie und Orthopädie

Warener Str. 7

12683 Berlin

Dr. med. Hubert Mayer

Chirurgische Gemeinschaftspraxis am Vincentinum

Franziskanergasse 14

86152 Augsburg

Dr. med. Marcus Raum

Univers. Med Centr Groningen

Aandachtsgebied Traumatologie, Afdeling Chirurgie W4.242

Postbus 3001

9700 Rb Groningen (Netherlands)

Prof. Dr. med. Eckhard Rickels

Allgemeines Krankenhaus Celle

Klinik für Unfallchirurgie, Orthopädie

und Neurotraumatologie

Siemensplatz 4

29223 Celle

PD Dr. med. Stefan Sauerland

Institut für Forschung in der Operativen Medizin

Universität Witten/Herdecke

Ostmerheimerstr. 200

51109 Köln

Dr. med. Ulrich Schächinger

Universitätsklinikum Regensburg

Abteilung für Unfallchirurgie

Franz-Josef-Strauss-Allee 11

93053 Regensburg

PD Dr. med. Michael Schädel-Höpfner

Universitätsklinikum Düsseldorf

Klinik für Unfall- und Handchirurgie

Moorenstraße 5

40225 Düsseldorf
Prof. Dr. med. Th. Schildhauer

Berufsgenossenschaftliches Universitätsklinikum

Bergmannsheil

Chirurgische Universitäts- und Poliklinik

Bürkle-de-la-Camp-Platz 1

44789 Bochum

PD Dr. med. Karsten Schwerdtfeger

Universitätsklinikum des Saarlandes

Klinik für Neurochirurgie

Kirrbergerstraße

66421 Homburg/Saar

Prof. Dr. med. Andreas Seekamp

Universitätsklinikum Schleswig-Holstein (Campus Kiel)

Klinik für Unfallchirurgie

Arnold-Heller-Str. 7

24105 Kiel

Dr. med. Erwin Stolpe

Gartenseeweg 8

82402 Seeshaupt

Prof. Dr. med. Johannes Sturm

Schlüterstr. 32

48149 Münster

PD Dr. med. Felix Walcher

Universitätsklinikum Frankfurt

Klinik für Unfall-, Hand- und Wiederherstellungschirurgie

Theodor-Stern-Kai 7

60590 Frankfurt

Prof. Dr. med. Christian Waydhas

Universitätsklinikum Essen

Klinik für Unfallchirurgie

Hufelandstr. 55

45147 Essen 


\section{Literatur}

\section{Einführende Kästen}

1 Council of Europe. Developing a methodology for drawing up guidelines on best medical practices: Recommendation Rec (2001) 13 adopted by the Committee of Ministers of the Council of Europe on 10 October 2001 and explanatory memorandum. 2001, Strasbourg Cedex: Council of Europe

2 Schmiegel W et al. S3-Leitlinie "Kolorektales Karzinom". Available from: www.krebsgesellschaft.de/download/ s3_Il_kolorektales_karzinom_2008.pdf

\section{Thoraxtrauma}

1a Ahmed-Nusrath A, Nusrath MA, Annamaneni R. Faecal mediastinitis following decompression of suspected tension pneumothorax. Emerg Med ] 2007; 24: 830 [LoE 5]

2a Ahmed MY, Silver P, Nimkoff L et al. The needle-wire-dilator technique for the insertion of chest tubes in pediatric patients. Pediatr Emerg Care 1995; 11: 252-254 [LoE 4]

3a Altman E, Ben-Nun A, Curtis WJ et al. Modified Seldinger technique for the insertion of standard chest tubes. Am J Surg 2001; 181: $354-355$ [LoE 5]

4a Andrabi SA, Andrabi SI, Mansha M et al. An iatrogenic complication of closed tube thoracostomy for penetrating chest trauma. N Z Med ] 2007; 120: U2784 [LoE 5]

5a Andrivet P, Djedaini K, Teboul J et al. Spontaneous pneumothorax: comparison of thoracic drainage vs. immediate or delayed needle aspiration. Chest 1995; 108: 335-338 [LoE 4]

6a Argall J, Desmond J. Seldinger technique chest drains and complication rate. Emerg Med J 2003; 20: 169-170 [LoE 1]

7a Aufmkolk M, Ruchholtz S, Hering $M$ et al. Wertigkeit der subjektiven Einschätzung der Thoraxverletzungsschwere durch den Notarzt. Unfallchirurg 2003; 106: 746-753 [LoE 4]

8a Aylwin CL, Brohi K, Davies GD et al. Pre-hospital and in-hospital thoracostomy: indications and complications. Ann R Coll Surg Engl 2008; 90: 54-57 [LoE 3]

9a Bailey RC. Complications of tube thoracostomy in trauma. J Accid Emerg Med 2000; 17: 111-114 [LoE 4]

10a Baldt M, Bankier A, Germann P et al. Complications after emergency tube thoracostomy: assessment with CT. Radiology 1995; 195: 539-543 [LoE 4]

11a Ball CG, Lord J, Laupland KB et al. Chest tube complications: how well are we training our residents? Can J Surg 2007; 50: 450-458 [LoE 2]

12a Barak M, laroshevski D, Ziser A. Rapid atrial fibrillation following tube thoracostomy insertion. Eur J Cardiothorac Surg 2003; 24: 461-462 [LoE 5]

13a Barton E. Tension pneumothorax. Curr Opin Pulm Med 1999; 5 : 269-274 [LoE 5]

14a Barton E, Epperson M, Hoyt D et al. Prehospital needle aspiration and tube thoracostomy in trauma victims: a six-year experience with aeromedical crews. J Emerg Med 1995; 13 : $155-163$ [LoE 4]

15a Bayne CG. Pulmonary complications of the McSwain Dart. Ann Emerg Med 1982; 11: 136-137 [LoE 5] 16a Beall jr. AC, Bricker DL, Crawford HW et al. Considerations in the management of penetrating thoracic trauma. J Trauma 1968; 8: $408-417$ [LoE 4]

17a Behnia MM, Garrett K. Association of tension pneumothorax with use of small-bore chest tubes in patients receiving mechanical ventilation. Crit Care Nurse 2004; 24: 64-65 [LoE 5]

18a Ben Ze'ev I. Percutaneous thoracostomy with plastic-shielded locking trocar. J Thorac Cardiovasc Surg 1995; 110: 273 [LoE 5]

19a Bergaminelli $C$, De Angelis $P$, Gauthier $P$ et al. Thoracic drainage in trauma emergencies. Minerva Chir 1999; 54: 697-702 [LoE 4]

20a Bernstein A, Waqaruddin M, Shah M. Management of spontaneous pneumothorax using a Heimlich flutter valve. Thorax 1973; 28: 386-389 [LoE 4]

21a Bertino RE, Wesbey GE, Johnson RJ. Horner syndrome occurring as a complication of chest tube placement. Radiology 1987; 164: 745 [LoE 5]

22a Biffl WL. Needle thoracostomy: a cautionary note. Acad Emerg Med 2004; 11: 795 -796, author reply 796 [LoE 5]

23a Blostein P, Hodgman C. Computed tomography of the chest in blunt thoracic trauma: results of a prospective study. J Trauma 1997; 43: $13-18$ [LoE 2]

24a Bokhari F, Brakenridge S, Nagy K et al. Prospective evaluation of the sensitivity of physical examination in chest trauma. J Trauma 2002; 53: 1135-1138 [LoE 2]

25a Brasel K, Stafford R, Weigelt J et al. Treatment of occult pneumothoraces from blunt trauma. J Trauma 1999; 46: 987-990 [LoE 1]

26a Bristol JB, Harvey JE. Safer insertion of pleural drains. Br Med J (Clin Res Ed) 1983; 286: 348-349 [LoE 5]

27a Britten S, Palmer SH. Chest wall thickness may limit adequate drainage of tension pneumothorax by needle thoracocentesis. J Accid Emerg Med 1996; 13: 426-427 [LoE 2]

28a Britten S, Palmer SH, Snow TM. Needle thoracocentesis in tension pneumothorax: insufficient cannula length and potential failure. Injury 1996; 27: 321 -322 [LoE 4]

29a Bushby N, Fitzgerald M, Cameron P et al. Prehospital intubation and chest decompression is associated with unexpected survival in major thoracic blunt trauma. Emerg Med Australas 2005; 17: 443 -449 [LoE 3]

30a Butler KL, Best IM, Weaver WL et al. Pulmonary artery injury and cardiac tamponade after needle decompression of a suspected tension pneumothorax. J Trauma 2003; 54: 610-611 [LoE 5]

31a Campbell P, Neil T, Wake PN. Horner's syndrome caused by an intercostal chest drain. Thorax 1989; 44: 305-306 [LoE 4]

32a Carney M, Ravin CE. Intercostal artery laceration during thoracocentesis: increased risk in elderly patients. Chest 1979; 75 : $520-522$ [LoE 4]

33a Casillas JA, De La Fuente A. Right atrium perforation by a pleura drain. Report of a case with survival. Thorac Cardiovasc Surg 1983; 31: 247-248 [LoE 4]

34a Chan L, Reilly KM, Henderson C et al. Complication rates of tube thoracostomy. Am J Emerg Med 1997; 15: 368 -370 [LoE 4]

35a Chen S, Chang K, Hsu C. Accuracy of auscultation in the detection of haemopneumothorax. Eur J Surg 1998; 164: 643-645 [LoE 1]

36a Chen S, Markmann J, Kauder D et al. Hemopneumothorax missed by auscultation in penetrating chest injury. J Trauma 1997; 42: 86-89 [LoE 3] 
37a Coats T, Wilson A, Xeropotamous N. Pre-hospital management of patients with severe thoracic injury. Injury 1995; 26: $581-585$ [LoE 4]

38a Collins J, Levine G, Waxman K. Occult traumatic pneumothorax: immediate tube thoracostomy versus expectant management. Am Surg 1992; 58: $743-746$ [LoE 4]

39a Committee on Trauma. Advanved Trauma Life Support (ATLS ${ }^{\circledR}$ ) - Reference Manual. American College of Surgeons, Chicago 2001 [LoE 5]

40a Committee on Trauma. Advanved Trauma Life Support (ATLS ${ }^{\circledR}$ ) - Reference Manual. American College of Surgeons, Chicago 1997 [LoE 5]

41a Conces jr. DJ, Tarver RD, Gray WC et al. Treatment of pneumothoraces utilizing small caliber chest tubes. Chest 1988; 94 : 55-57 [LoE 4]

42a Cooper C, Hardcastle T. Xpand chest drain: assessing equivalence to current standard therapy - a randomised controlled trial. S Afr J Surg 2006; 44: 131-135 [LoE 1]

43a Cox PA, Keshishian JM, Blades BB. Traumatic arteriovenous fistula of the chest wall and lung. Secondary to insertion of an intercostal catheter. J Thorac Cardiovasc Surg 1967; 54: 109-112 [LoE 4]

44a Cullinane D, Morris J, Bass J et al. Needle thoracostomy may not be indicated in the trauma patient. Injury 2001; 32: 749-752 [LoE 4]

45a Curtin J, Goodman L, Quebbeman E et al. Thoracostomy tubes after acute chest injury: relationship between location in a pleural fissure and function. AJR 1994; 163: 1339-1342 [LoE 4]

46a Daly RC, Mucha P, Pairolero PC et al. The risk of percutaneous chest tube thoracostomy for blunt thoracic trauma. Ann Emerg Med 1985; 14: 865-870 [LoE 4]

47a David A. Thoraxsaugdrainagen bei der Erstversorgung von Brustkorbverletzungen. Notfallmed 1985; 11: 1481 [LoE 4]

48a Davis DP, Pettit K, Rom CD et al. The safety and efficacy of prehospital needle and tube thoracostomy by aeromedical personnel. Prehosp Emerg Care 2005; 9: 191 -197 [LoE 2]

49a De La Fuente A, Sanchez R, Suarez J et al. Left ventricular perforation by a pleural drainage tube. Report of a case with survival. Tex Heart Inst ] 1994; 21: 175-176 [LoE 4]

50a Deakin C, Davies G, Wilson A. Simple thoracostomy avoids chest drain insertion in prehospital trauma. J Trauma 1995; 39 : 373-374 [LoE 4]

51a Delius R, Obed F, Horst $M$ et al. Catheter aspiration for simple pneumothorax. Arch Surg 1989; 124: 833 -836 [LoE 3]

52a Demartines N, Kiener A, Scheidegger D et al. [Thoracic drainage at the accident site]. Helv Chir Acta 1990; 57: 273-277 [LoE 4]

53a Demartines N, Kiener A, Scheidegger D et al. [Thoracic drainage at the accident site]. Helv Chir Acta 1990; 57: 273-277 [LoE 4]

54a Deneuville M. Morbidity of percutaneous tube thoracostomy in trauma patients. Eur J Cardiothorac Surg 2002; 22: 673-678 [LoE 2]

55a Di Bartolomeo S, Sanson G, Nardi G et al. A population-based study on pneumothorax in severely traumatized patients. J Trauma 2001; 51: 677-682 [LoE 4]

56a Dominguez Fernandez E, Neudeck F, Piotrowski ]. [Perforation of the heart wall - a rare complication after thoracic drainage treatment]. Chirurg 1995; 66: 920-922 [LoE 4] 57a Duponselle EF. The level of the intercostal drain and other determinant factors in the conservative approach to penetrating chest injuries. Cent Afr ] Med 1980; 26: 52 -55 [LoE 4]

58a Eckstein M, Suyehara D. Needle thoracostomy in the prehospital setting. Prehosp Emerg Care 1998; 2: 132-135 [LoE 2]

59a Eddy A, Luna G, Copass M. Empyema thoracis in patients undergoing emergent closed tube thoracostomy for thoracic trauma. Am J Surg 1989; 157: 494-497 [LoE 4]

60a Enderson B, Abdalla R, Frame $S$ et al. Tube thoracostomy for occult pneumothorax: a prospective randomized study of its use. J Trauma 1993; 35: 726-729 [LoE 1]

61a Eriksson A. Fatal iatrogenic exsanguination from pleural drain insertion into the inferior cava. Thorac Cardiovasc Surg 1982; 30: 191 - 193 [LoE 4]

62a Etoch S, Bar-Natan M, Miller F et al. Tube thoracostomy. Factors related to complications. Arch Surg 1995; 130: 521-525 [LoE 4]

63a Fitzgerald M, Mackenzie CF, Marasco S et al. Pleural decompression and drainage during trauma reception and resuscitation. Injury 2008; 39: 9-20 [LoE 5]

64a Foresti V, Villa A, Casati O et al. Abdominal placement of tube thoracostomy due to lack of recognition of paralysis of hemidiaphragm. Chest 1992; 102: 292-293 [LoE 5]

65a Fraser RS. Lung perforation complicating tube thoracostomy: pathologic description of three cases. Hum Pathol 1988; 19 : 518-523 [LoE 4]

66a Gaillard M, Herve C, Mandin L et al. Mortality prognostic factors in chest injury. J Trauma 1990; 30: 93-96 [LoE 3]

67a Galloway P, King P, Freeland P et al. Use of autosuture surgiport for pleural drain insertion. Injury 1993; 24: 538-540 [LoE 4]

68a Gammie JS, Banks MC, Fuhrman CR et al. The pigtail catheter for pleural drainage: a less invasive alternative to tube thoracostomy. JSLS 1999; 3: 57-61 [LoE 4]

69a Gammie JS, Banks MC, Fuhrman CR et al. The pigtail catheter for pleural drainage: a less invasive alternative to tube thoracostomy. JSLS1999; 3: 57-61 [LoE 4]

70a Garramone RJ, Jacobs L, Sahdev P. An objective method to measure and manage occult pneumothorax. Surg Gynecol Obstet 1991; 173: 257-261 [LoE 4]

71a Gill S, Nkere U, Walesby R. An expansible cannula: a new technique for chest drain insertion. Thorax 1992; 47: 657-659 [LoE 4]

72a Givens M, Ayotte K, Manifold C. Needle thoracostomy: implications of computed tomography chest wall thickness. Acad Emerg Med 2004; 11: 211 -313 [LoE 3]

73a Graham AN, Cosgrove AP, Gibbons JR et al. Randomised clinical trial of chest drainage systems. Thorax 1992; 47: 461-462 [LoE 2]

74a Harcke HT, Pearse LA, Levy AD et al. Chest wall thickness in military personnel: implications for needle thoracentesis in tension pneumothorax. Mil Med 2007; 172: 1260-1263 [LoE 2]

75a Heim P, Maas R, Tesch C et al. [Pleural drainage in acute thoracic trauma. Comparison of the radiologic image and computer tomography]. Aktuelle Radiol 1998; 8: 163-168 [LoE 4]

76a Helling T, Gyles N, Eisenstein C et al. Complications following blunt and penetrating injuries in 216 victims of chest trauma requiring tube thoracostomy. J Trauma 1989; 29: 1367-1370 [LoE 4] 
77a Heng K, Bystrzycki A, Fitzgerald M et al. Complications of intercostal catheter insertion using EMST techniques for chest trauma. ANZ ] Surg 2004; 74: 420-423 [LoE 4]

78a Hiebl A. Problematik von Heimlich-Ventilen in der Notfallversorgung. Medizinische Fakultät der Ludwig-Maximilians-Universität, München: Medizinische Fakultät der LMU; 2001 [LoE 4]

79a Hing A, Caldwell E, Nguyen T et al. Pre-hospital care of gun shot and stabbing injuries: an evaluation of practice and clinical outcome. Pre-hospital Immediate Care 2001; 5: 6-11 [LoE 4]

80a Hirshberg A, Thomson S, Huizinga W. Reliability of physical examination in penetrating chest injuries. Injury 1988; 19: 407-409 [LoE 1]

81a Hostetler MA, Davis CO. Bilateral localized tension pneumothoraces refractory to needle decompression. Pediatr Emerg Care 1999; 15: 322-324 [LoE 4]

82a Huber-Wagner S, Korner M, Ehrt A et al. Emergency chest tube placement in trauma care - which approach is preferable? Resuscitation 2007; 72: 226-233 [LoE 2]

83a Hyde J, Sykes T, Graham T. Reducing morbidity from chest drains. BM] 1997; 314: 914-915 [LoE 5 ]

84a Jenkins $C$, Sudheer P. Needle thoracocentesis fails to diagnose a large pneumothorax. Anaesthesia 2000; 55: 925-926 [LoE 4]

85a Johnson G. Traumatic pneumothorax: is a chest drain always necessary? J Accid Emerg Med 1996; 13: 173-174 [LoE 4]

86a Kang SS. Use of the disposable laparoscopic trocar-cannula for chest tube insertion. J Am Coll Surg 1994; 179: 230 [LoE 5]

87a Kirkpatrick AW. Clinician-performed focused sonography for the resuscitation of trauma. Crit Care Med 2007; 35: S162-172 [LoE 5]

88a Lechleuthner A, Bouillon B, Neugebauer E et al. Prehospital chest tubes - incidence and analysis of iatrogenic injuries in the emergency medical service in Cologne. Theor Surg 1994; 9 : 220-226 [LoE 4]

89a Lee C, Revell M, Porter K et al. The prehospital management of chest injuries: a consensus statement. Faculty of Pre-hospital Care, Royal College of Surgeons of Edinburgh. Emerg Med ] 2007; 24: 220-224 [LoE 5]

90a Leigh-Smith S, Davies G. Tension pneumothorax: eyes may be more diagnostic than ears. Emerg Med J 2003; 20: 495-496 [LoE 5]

91a Leigh-Smith S, Harris T. Tension pneumothorax - time for a re-think. Emerg Med ] 2005; 22: 8-16 [LoE 1]

92a Lyass S, Simha M, Muggia-Sullam M. Use of a laparoscopic trocar for tube thoracostomy-a rapid and safe method for chest tube placement. Eur ] Emerg Med 1995; 2: 96 [LoE 5]

93a Mainini SE, Johnson FE. Tension pneumothorax complicating small - caliber chest tube insertion. Chest 1990; 97: 759-760 [LoE 4]

94a Mandal A, Thadepalli H, Mandal A et al. Posttraumatic empyema thoracis: a 24-year experience at a major trauma center. J Trauma 1997; 43: 764-771 [LoE 5]

95a Marinaro JL, Kenny CV, Smith SR et al. Needle thoracostomy in trauma patients: what catheter length is adequate? Acad Emerg Med 2003; 10: 495 [LoE 3]

96a Martin T, Fontana G, Olak J et al. Use of pleural catheter for the management of simple pneumothorax. Chest 1996; 110: 1169-1172 [LoE 5]

97a Massarutti D, Trillo G, Berlot G et al. Simple thoracostomy in prehospital trauma management is safe and effective: a 2-year experience by helicopter emergency medical crews. Eur J Emerg Med 2006; 13: 276 -280 [LoE 2]

98a McConaghy PM, Kennedy N. Tension pneumothorax due to intrapulmonary placement of intercostal chest drain. Anaesth Intensive Care 1995; 23: 496-498 [LoE 4]

99a Mcintosh N, Becher Jc, Cunningham S et al. Clinical diagnosis of pneumothorax is late: use of trend data and decision support might allow preclinical detection. Pediatr Res 2000; 48: 408-415 [LoE 4]

100a Mcpherson J], Feigin DS, Bellamy RF. Prevalence of tension pneumothorax in fatally wounded combat casualties. J Trauma 2006; 60: 573-578 [LoE 2]

101a Mcroberts R, Mckechnie M, Leigh-Smith S. Tension pneumothorax and the "forbidden CXR". Emerg Med J 2005; 22: 597-598 [LoE 5]

102a McSwain jr. NE. The McSwain Dart: device for relief of tension pneumothorax. Med Instrum 1982; 16: 249-250 [LoE 5]

103a McSwain jr. NE. A thoracostomy tube for field and emergency department use. JACEP 1997; 6: 324-325 [LoE 4]

104a Meisel S, Ram Z, Priel I et al. Another complication of thoracostomy - perforation of the right atrium. Chest 1990; 98 : $772-773$ [LoE 4]

105a Melamed E, Blumenfeld A, Lin G. Locking plastic tie - a simple technique for securing a chest tube. Prehosp Disaster Med 2007; 22: 344-345 [LoE 5]

106a Mellor DJ. A new method of chest drain insertion. Anaesthesia 1996; 51: $713-714$ [LoE 5]

107a Millikan JS, Moore EE, Steiner E et al. Complications of tube thoracostomy for acute trauma. Am J Surg 1980; 140: $738-741$ [LoE 4]

108a Mines D, Abbuhl S. Needle thoracostomy fails to detect a fatal tension pneumothorax. Ann Emerg Med 1993; 22: 863-866 [LoE 4]

109a Moskal T, Liscum K, Mattox K. Subclavian artery obstruction by tube thoracostomy. J Trauma 1997; 43: 368-369 [LoE 4]

110a Netto FA, Shulman H, Rizoli SB et al. Are needle decompressions for tension pneumothoraces being performed appropriately for appropriate indications? Am J Emerg Med 2008; 26 : 597-602 [LoE 2]

111a Niemi T, Hannukainen J, Aarnio P. Use of the Heimlich valve for treating pneumothorax. Ann Chir Gynaecol 1999; 88: 36-37 [LoE 2]

112a Noppen M, Alexander P, Driesen P et al. Manual aspiration versus chest tube drainage in first episodes of primary spontaneous pneumothorax. Am J Respir Crit Care Med 2002; 165 : $1240-1244$ [LoE 4]

113a Nosher Jl, Siegel R. Over-the-wire placement of large bore thoracostomy tubes. Cardiovasc Intervent Radiol 1993; 16: 195-197 [LoE 4]

114a Pattison GT. Needle thoracocentesis in tension pneumothorax: insufficient cannula length and potential failure. Injury 1996; 27: 758 [LoE 4]

115a Peek G, Firmin R. Reducing morbidity from insertion of chest drains. Br Med J 1997; 315: 313 [LoE 5]

116a Peek G], Firmin RK, Arsiwala S. Chest tube insertion in the ventilated patient. Injury 1995; 26: 425-426 [LoE 4]

117a Peters S, Wolter D, Schultz ]. [Dangers and risks of thoracic drainage at the accident site]. Unfallchirurg 1996; 99: 953-957 [LoE 4] 
118a Rashid M, Acker A. Cardiac herniation with catheterization of the heart, inferior vena cava, and hepatic vein by a chest tube. J Trauma 1998; 45: 407-409 [LoE 4]

119a Rawlins R, Brown KM, Carr CS et al. Life threatening haemorrhage after anterior needle aspiration of pneumothoraces. A role for lateral needle aspiration in emergency decompression of spontaneous pneumothorax. Emerg Med J 2003; 20: 383-384 [LoE 5]

120a Remerand $F$, Luce $V$, Badachi $Y$ et al. Incidence of chest tube malposition in the critically ill: a prospective computed tomography study. Anesthesiology 2007; 106: 1112-1119 [LoE 2]

121a Roberts J, Bratton S, Brogan T. Efficacy and complications of percutaneous pigtail catheters for thoracostomy in pediatric patients. Chest 1998; 114: 1116-1121 [LoE 4]

122a Röggla $M$, Wagner $A$, Brunner $C$ et al. The management of pneumothorax with the thoracic vent versus conventional intercostal tube drainage. Wien Klin Wochenschr 1996; 108: 330-333 [LoE 2]

123a Rüter A, Trentz O, Wagner M. Thorax-Akuttherapie: Minithorakotomie als empfohlenes Vorgehen. In: Rüter A, Trentz O, Wagner M, Hrsg. Unfallchirurgie. Urban \& Schwarzenberg, München Wien Baltimore 1995; 315-316 [LoE 5]

124a Rutherford RB, Hurt jr. HH, Brickman RD et al. The pathophysiology of progressive, tension pneumothorax. J Trauma 1968; 8: 212-227 [LoE 5]

125a Schmidt U, Stalp M, Gerich T et al. Chest tube decompression of blunt chest injuries by physicians in the field: effectiveness and complications. J Trauma 1998; 44: $98-100$ [LoE 4]

126a Schöchl H. Präklinische Versorgung des schweren Thoraxtraumas. Notfallmedizin 1994; 6: 310 [LoE 4]

127a Shih C, Chang Y, Lai S. Successful management of perforating injury of right atrium by chest tube. Chung Hua I Hsueh Tsa Chih 1992; 50: 338-340 [LoE 4]

128a Spanjersberg W, Ringburg A, Bergs B et al. Prehospital chest tube thoracostomy: effective treatment or additional trauma? J Trauma 2005; 59: 96-101 [LoE 2]

129a Sriussadaporn S, Poomsuwan P. Post-traumatic empyema thoracis in blunt chest trauma. J Med Assoc Thai 1995; 78: 393-398 [LoE 4]

130a Steier M, Ching N, Roberts EB et al. Pneumothorax complicating continuous ventilatory support. J Thorac Cardiovasc Surg 1974; 67: 17-23

131a Subotich D, Mandarich D. Accidentally created tension pneumothorax in patient with primary spontaneous pneumothoraxconfirmation of the experimental studies, putting into question the classical explanation. Med Hypotheses 2005; 64: 170-173 [LoE 5]

132a Symbas P. Chest drainage tubes. Surg Clin N Am 1989; 69: 41-46 [LoE 5]

133a Tang A, Hooper T, Hasan R. A regional survey of chest drains: evidence-based practice? Postgrad Med] 1999; 75:471 -474 [LoE5]

134a Thal AP, Quick KL. A guided chest tube for safe thoracostomy. Surg Gynecol Obstet 1988; 167: 517 [LoE 5]

135a Thomson S, Huizinga W, Hirshberg A. Prospective study of the yield of physical examination compared with chest radiography in penetrating thoracic trauma. Thorax 1990; 45: 616-619 [LoE 1]

136a Tomlinson MA, Treasure T. Insertion of a chest drain: how to do it. Br] Hosp Med 1997; 58: 248-252 [LoE 5] 137a Trupka A, Waydhas C, Hallfeldt KKF et al. The value of thoracic computed tomography in the first assessment of severely injured patients with blunt chest trauma. J Trauma 1997; 43: 405-411 [LoE 2]

138a Velanovich $V$, Adams $C$. The use of colostomy bags for chest tube drainage. Ann Thorac Surg 1988; 46: 697-698 [LoE 5]

139a Velez SE, Sarquis G. [Utility of digital thoracotomy in chest trauma]. Rev Fac Cien Med Univ Nac Cordoba 2006; 63: 7-10 [LoE 3]

140a Waksman I, Bickel A, Szabo A et al. Use of endoscopic trocarcannula for chest drain insertion in trauma patients and others. J Trauma 1999; 46: 941-943 [LoE 4]

141a Wayne MA, McSwain jr. NE. Clinical evaluation of a new device for the treatment of tension pneumothorax. Ann Surg 1980; 191: 760-762 [LoE 4]

142a Williams JG, Riley TR, Moody RA. Resuscitation experience in the Falkland Islands campaign. Br Med J (Clin Res Ed) 1983; 286: 775-777 [LoE 4]

143a Wormald $P$, Knottenbelt J, Linegar A. A triage system for stab wounds to the chest. S Afr Med J 1989; 76: 211-212 [LoE 3]

144a York D, Dudek L, Larson $R$ et al. A comparison study of chest tube thoracostomy: air medical crew and in-hospital trauma service. Air Med J 1993; 12: 227-229

145a Zengerink I, Brink PR, Laupland KB et al. Needle thoracostomy in the treatment of a tension pneumothorax in trauma patients: what size needle? J Trauma 2008; 64: 111-114 [LoE 2]

\section{Schädel-Hirn-Trauma}

1b Alderson P, Roberts I. Corticosteroids for acute traumatic brain injury. Cochrane Database Syst Rev 2005; 1: CD 000196

2b Balestreri M, Czosnyka M, Chatfield DA et al. Predictive value of Glasgow Coma Scale after brain trauma: change in trend over the past ten years. J Neurol Neurosurg Psychiatry 2004; 75 : $161-162$

3b Brihaye J, Frowein RA, Lindgren S et al. Report on the meeting of the WFNS Neuro-Traumatology Committee. Brussels. I. Coma scaling. Acta Neurochir (Wien) 1978; 40: 181-186

4b CRASH trial collaborators. Effect of intravenous corticosteroids on death within 14 days in 10008 adults with clinically significant head injury (MRC CRASH trial): randomised placebo-controlled trial. Lancet 2004; 364: 1321-1328

5b CRASH trial collaborators. Final results of MRC CRASH, a randomised placebo-controlled trial of intravenous corticosteroid in adults with head injury - outcomes at 6 months. Lancet 2005; 365: 1957-1959 [LoE 1b]

6b Firsching R, Messing-Jünger M, Rickels E et al. Leitlinie Schädelhirntrauma im Erwachsenenalter der Deutschen Gesellschaft für Neurochirurgie. AWMF online 2007. Im Internet: www. uni-duesseldorf.de/AWMF/II/008-001.htm

7b Frowein RA. Classification of coma. Acta Neurochir 1976; 34: 5-10

8b Gabriel EJ, Ghajar J, Jagoda A et al.; Brain Trauma Foundation. Guidelines for prehospital management of traumatic brain injury. ] Neurotrauma 2002; 19: 111 -174 [Evidenzbasierte Leitlinie]

9b Gurdjian ES, Brihaye J, Christensen JC et al. Glossary of Neurotraumatology. Acta Neurochir (Wien) Suppl. 25. Wien, New York: Springer; 1979 
10b Karimi A, Burchardi H. Deutsche Interdisziplinäre Vereinigung für Intensiv- und Notfallmedizin (DIVI) Stellungnahmen, Empfehlungen zu Problemen der Intensiv- und Notfallmedizin. 5. Auflage. Köln: asmuth druck + crossmedia; 2004

11b Langham J, Goldfrad C, Teasdale G et al. Calcium channel blockers for acute traumatic brain injury (Cochrane Review). The Cochrane Library, Issue 1, John Wiley \& Sons, Ltd., Chichester 2004

12b Lorenz R. Neurotraumatologie. Standardisierte Nomenklatur. Springer, Berlin 1990

13b Kraus JF, Black MA, Hessol $\mathrm{N}$ et al. The incidence of acute brain injury and serious impairment in a defined population. Am J Epidemiol 1984; 119: 186-201

14b Kraus JF, Fife D, Conroy C. Incidence, severity, and outcomes of brain injuries involving bicycles. Am J Public Health 1987; 77: $76-78$

15b Maas $A$ et al. EBIC-Guidelines for mangement of severe head injury in adults. Acta Neurchir (Wien) 1997; 139: 286-294 [Evidenzbasierte Leitlinie]

16b Marion DW, Carlier PM. Problems with initial Glasgow Coma Scale assessment caused by prehospital treatment of patients with head injuries: results of a national survey. J Trauma 1994; 36: 89-95

17b Marmarou A, Lu J, Butcher I et al. Prognostic value of the Glasgow Coma Scale and pupil reactivity in traumatic brain injury assessed pre-hospital and on enrollment: an IMPACT analysis. J Neurotrauma 2007; 24: 270-280 [LoE 3a]

18b Roberts I. Aminosteroids for acute traumatic brain injury. (Cochrane Review) In: The Cochrane Library, Issue 1, John Wiley \& Sons, Ltd., Chichester 2004

19b Roberts I. Barbiturates for acute traumatic brain injury. (Cochrane Review) In: The Cochrane Library, Issue 1, John Wiley \& Sons, Ltd., Chichester 2004

20b Schierhout G, Roberts I. Anti-epileptic drugs for preventing seizures following acute traumatic brain injury. (Cochrane Review) In: The Cochrane Library, Issue 1, John Wiley \& Sons, Ltd., Chichester 2004

21b Teasdale G, Jennett B. Assessment of coma and impaired consciousness. A practical scale.. Lancet 1974; 2: 81-84

22b Teasdale $G$, Jennett $B$. Assessment and prognosis of coma after head injury. Acta Neurochir (Wien) 1976; 34: 45-55

23b The Brain Trauma Foundation; The American Association of Neurological Surgeons; The Joint Section on Neurotrauma and Critical Care. Management and Prognosis of Severe Traumatic Brain Injury. 2000. Im Internet: www2.braintrauma.org/ guidelines/downloads/btf_guidelines_management.pdf [Evidenzbasierte Leitlinie]

24 The Brain Trauma Foundation; The American Association of Neurological Surgeons; The Joint Section on Neurotrauma and Critical Care. Management and Prognosis of Severe Traumatic Brain Injury. Update 2003. Im Internet: www2.braintrauma. org/guidelines/downloads/btf_guidelines_cpp_u1.pdf [Evidenzbasierte Leitlinie]

25 The Brain Trauma Foundation; The American Association of Neurological Surgeons; The Joint Section on Neurotrauma and Critical Care. Guidelines for the Management of Severe Traumatic Brain Injury. 3rd ed. Im Internet: www.braintrauma.org/ guidelines/downloads/JON_24_Supp1.pdf [Evidenzbasierte Leitlinie] 26b Tien HC, Cunha JR, Wu SN et al. Do trauma patients with a Glasgow Coma Scale score of 3 and bilateral fixed and dilated pupils have any chance of survival? J Trauma 2006; 60: 274-278 [LoE 3b]

27b Tönnis W, Loew F. Einteilung der gedeckten Hirnschädigungen. Ärztliche Praxis 1953; 5: 13-14

28b Wakai A, Roberts IG, Schierhout G. Mannitol for acute traumatic brain injury. Cochrane Database Syst Rev 2007; 1: CD 001049 [LoE 3b]

29b Willis C, Lybrand S, Bellamy N. Excitatory amino acid inhibitors for traumatic brain injury. (Cochrane Review) In: The Cochrane Library, Issue 1, John Wiley \& Sons, Ltd., Chichester 2004

\section{Wirbelsäulenverletzungen}

1c Amar PA, Levy ML. Pathogenesis and pharmacological strategies for mitigatin secondary damage in acute spinal cord injury. Neurosurgery 1999; 44: 1027-1040

2c American College of Surgeons. Advanced Trauma Life Support. Chicago 1997

3c Asamoto S, Sugiyama $\mathrm{H}$, lida $\mathrm{M}$ et al. Trauma sites and clinical features associated with acute hyperextension spinal cord injury without bone damage-relationship between trauma site and severity. Neurol Med Chir (Tokyo) 2001; 41: 1-6; discussion 6-7

4c Asazuma T, Satomi K, Suzuki N et al. Management of patients with an incomplete cervical spinal cord injury. Spinal Cord 1996; 34: 620-625

5c Bandiera G, Stiell IG, Wells GA et al. The Canadian c-spine rule performs better than unstructured physician judgment. Ann Emerg Med 2003; 42: 395-402

6c Beck A, Krischak G, Bischoff M. Wirbelsäulenverletzungen und spinales Trauma. Notfall Rettungsmed 2009; 12: 469-479

7c Beck A, Gebhard F, Kinzl L et al. Spinal cord injury without radiographic abnormalities in children and adolescents. Knee Surg, Sports Traumatol Arthrosc 2000; 8: 186-189

8c Beck A, Gebhard F, Kinzl L et al. Prinzipien und Techniken der unfallchirurgischen Erstversorgung am Einsatzort. Unfallchirurg 2001; 104: 1082 -1099

9c Blackmore C, Emerson S, Mann F et al. Cervical spine imaging in patients with trauma: Determination of fracture risk to optimize use. Radiology 1999; 211: 759-766

10c Bracken MB, Holford TR. Effects of timing of methylprednisolone or naloxone administration on recovery of segmental and long-tract neurological function in NASCIS2. J Neurosurg 1993; 79: $500-507$

11c Bracken MB, Shepard MJ, Collins WF et al. A randomized, controlled trial of methylprednisolone or naloxone in the treatment of acute spinal-cord injury. Results of the Second National Acute Spinal Cord Injury Study. N Engl J Med 1990; 322: 1405 1411

12c Bracken MB, Shepard MJ, Collins jr. WF et al. Methylprednisolone or naloxone treatment after acute spinal cord injury: 1-year follow-up data. Results of the second National Acute Spinal Cord Injury Study. J Neurosurg 1992; 76: 23-31

13c Bracken MB, Shepard MJ, Hellenbrand KG et al. Methylprednisolone and neurological function 1 year after spinal cord injury. Results of the National Acute Spinal Cord Injury Study. J Neurosurg 1985; 63: 704-713 
14c Bracken MB, Shepard MJ, Holford TR et al. Administration of methylprednisolone for 24 or 48 hours or tirilazad mesylate for 48 hours in the treatment of acute spinal cord injury. Results of the Third National Acute Spinal Cord Injury Randomized Controlled Trial. National Acute Spinal Cord Injury Study. JAMA 1997; 277: 1597-1604

15c Bracken MB, Shepard MJ, Holford TR et al. Methylprednisolone or tirilazad mesylate administration after acute spinal cord injury: 1-year follow up. Results of the third National Acute Spinal Cord Injury randomized controlled trial. J Neurosurg 1998; 89: 699-706

16c Chandler DR, Nemejc C, Adkins RH et al. Emergency cervicalspine immobilization. Ann Emerg Med 1992; 21: 1185-1188

17c Chen XY, Carp JS, Chen L et al. Corticospinal tract transection prevents operantly conditioned $\mathrm{H}$-reflex increase in rats. Exp Brain Res 2002; 144: 88-94

18c Cline JR, Scheidel E, Bigsby EF. A comparison of methods of cervical immobilization used in patent extrication and transport. J Trauma 1985; 25: 649-653

19c Coleman WP, Benzel D, Cahill DW et al. A critical appraisal of the reporting of the National Acute Spinal Cord Injury Studies (II and III) of methylprednisolone in acute spinal cord injury. J Spinal Disord 2000; 13: 185-199

20c Cooper C, Dunham CM, Rodriguez A. Falls and major injuries are risk factors for thoracolumbar fractures: cognitive impairment and multiple injuries impede the detection of back pain and tenderness. J Trauma 1995; 38: 692-696

21c Craig G, Nielsen MS. Rigid cervical collars and intracranial pressure. Intensive Care Med 1991; 17: 504-505

22c Davies G, Deakin C, Wilson A. The effects of a rigid collar on intracranial pressure. Injury 1996; 27: 647-649

23c Domeier RM, Evans RW, Swor RA et al. Prospective validation of out-of-hospital spinal clearance criteria: a preliminary report. Acad Emerg Med 1997; 4: 643-646 [LoE 1a]

24c Domeier RM, Swor RA, Evans RW et al. Multicenter prospective validation of prehospital clinical spinal clearance criteria. J Trauma 2002; 53: 744-750

25c Ducker TB. Treatment of spinal-cord injury. N Engl J Med 1990; 322: $1459-1461$

26c Ducker TB, Zeidman SM. Spinal cord injury: Role of steroid therapy. Spine 1994; 19: 2281-2287

27c Epstein N, Hood DC, Ransohoff J. Gastrointestinal bleeding in patients with spinal cord trauma. Effects of steroids, cimetidine, and mini-dose heparin. J Neurosurg 1981; 54: 16-20

28c Frankel H, Rozycki G, Ochsner MG et al. Indication for obtaining surveillance thoracic and lumbar spine radiographs. J Trauma 1994; 37: 673-676

29c Galandiuk S, Raque G, Appel S et al. The two-edged sword of large-dose steroids for spinal cord trauma. Ann Surg 1993; 218: 419-427

30c George ER, Scholten DJ, Buechler CM et al. Failure of methylprednisolone to improve the outcome of spinal cord injuries. Am Surg 1995; 61: 659-664

31c Gerndt SJ, Rodriguez JL, Pawlik JW et al. Consequences of highdose steroid therapy for acute spinal cord injury. J Trauma 1997; 42: 279-284

32c Gertzbein SD. Neurologic deterioration in patients with thoracic and lumbar fractures after admission to the hospital. Spine 1994; 19: 1723-1725 33c Hanson JA, Blackmore CC, Mann FA et al. Cervical spine injury: a clinical decision rule to identify high-risk patients for helical CT screening. AJR Am J Roentgenol 2000; 174: 713-717

34c Ho AM-H, Fung KY et al. Rigid cervical collar and intracranial pressure of patients with severe head injury. J Trauma 2002; 53: $1185-1188$

35c Hoffman JR, Mower WR, Wolfson AB et al. Validity of a set of clinical criteria to rule out injury to the cervical spine in patients with blunt trauma. National Emergency X-Radiography Utilization Study Group. New Engl J Med 2000; 343: 94 -99

36c Holmes JF, Panacek EA, Miller PQ et al. Prospective evaluation of criteria for obtaining thoracolumbar radiographs in trauma patients. J Emerg Med 2003; 24: 1-7

37c Hunt K, Hallworth S, Smith M. The effects of rigid collar placement on intracranial and cerebral perfusion pressures. Anaesthesia 2001; 56: 511-513

38c Kolb JC, Summers RL, Galli RL. Cervical collar-induced changes in intracranial pressure. Am J Emerg Med 1999; 17: 135-137

39c Kuhnigk H, Bomke S, Sefrin P. [Effect of external cervical spine immobilization on intracranial pressure]. Aktuelle Traumatol 1993; 23: 350-353

40c Kwan I, Bunn F, Roberts I, Committee obotWP-HTC. Spinal immobilisation for trauma patients. The Cochrane Library Issue 4: Update Software 2001

41c Lam AM. Spinal cord injury: management. Curr Opin Anesth 1992; 5: 632-639

42c Matsumoto T, Tamaki T, Kawakami M et al. Early complications of high-dose methylprednisolone sodium succinate treatment in the follow-up of acute cervical spinal cord injury. Spine 2001; 26: $426-430$

43c Meldon SW, Moettus LN. Thoracolumbar spine fractures: clinical presentation and the effect of altered sensorium and major injury. J Trauma 1995; 39: 1110-1114 [LoE 4]

44c Mirza SK, Krengel WF 3rd, Chapman JR et al. Early versus delayed surgery for acute cervical spinal cord injury. Clin Orthop 1999; 359: 104-114 [LoE 4]

45c Muhr MD, Seabrook DL, Wittwer LK. Paramedic use of a spinal injury clearance algorithm reduces spinal immobilization in the out-of-hospital setting. Prehosp Emerg Care 1999; 3: 1 -6 [LoE 1a]

46c Nechwatal E. [Critical notes on the transport of patients with cervical spinal cord injuries]. Chirurg 1975; 46: 521-523

47c Nockels RP. Nonoperative management of acute spinal cord injury. Spine 2001; 26: S31-S37

48c Pointillart V, Petitjean ME, Wiart L et al. Pharmacological therapy of spinal cord injury during the acute phase. Spinal Cord 2000; 38: 71-76

49c Prendergast MR, Saxe JM, Ledgerwood AM et al. Massive steroids do not reduce the zone of injury after penetrating spinal cord injury. J Trauma 1994; 37: 576-579; discussion 579-580

50c Raphael J, Chotai R. Effects of the cervical collar on cerebrospinal fluid pressure. Anaesthesia 1994; 49: 437-439

51c Rosen PB, McSwain NE, Arata M et al. Comparison of two new immobilization collars. Ann Emerg Med 1992; 21: 1189-1195

52c Rosenfeld JF, Vaccaro AR, Albert TJ et al. The benefits of early decompression in cervical spinal cord injury. Am J Orthop 1998; 27: $23-28$

53c Ross SE, O'Malley KF, DeLong WG et al. Clinical predictors of unstable cervical spinal injury in multiply injured patients. Injury 1992; 23: 317-319 [LoE 2b] 
54c Sackett DL, Richardson WS, Rosenberg W et al. Evidence-based medicine: How to practice and teach EBM. Churchill Livingstone, London 1997

55c Sneed RC, Stover SL. Undiagnosed spinal cord injuries in braininjured children. Am J Dis Child 1988; 142: 965-967

56c Stiell IG, Wells GA, Vandemheen KL et al. The Canadian c-spine rule for radiopraphy in alert and stable trauma patients. JAMA 2001; 286: $1841-1848$

57c Sung RD, Wang JC. Correlation between a positive Hoffmann's reflex and cervical pathology in asymptomatic individuals. Spine 2001; 26: 67-70

58c Tator $\mathrm{CH}$. Experimental and clinical studies of the pathophysiology and management of acute spinal cord injury. J Spinal Cord Med 1996; 19: 206-214

59c Tator $\mathrm{CH}$. Biology of neurological recovery and functional restoration after spinal cord injury. Neurosurgery 1998; 42: $696-708$

60c Tator CH, Duncan EG, Edmonds VE et al. Neurological recovery, mortality and length of stay after acute spinal cord injury associated with changes in management. Paraplegia 1995; 33 : 254-262

61c Vogel P. [Neurologic disorders after injuries of the spine]. Langenbecks Arch Chir Suppl Kongressbd 1992: 271-273

62 Weinstein DE, Ko HY, Graziani V et al. Prognostic significance of the delayed plantar reflex following spinal cord injury. J Spinal Cord Med 1997; 20: 207-211

63c Wells JD, Nicosia S. Scoring acute spinal cord injury: a study of the utility and limitations of five different grading systems. J Spinal Cord Med 1995; 18: 33-41

64c Wing PC, Nance P, Connell DG et al. Risk of avascular necrosis following short term megadose methylprednisolone treatment. Spinal Cord 1998; 36: 633-636

65c Young W. Secondary injury mechanisms in acute spinal cord injury. J Emerg Med 1993; 11 (Suppl 1): 13-22

66c Zeidman SM, Ling GS, Ducker TB et al. Clinical applications of pharmacologic therapies for spinal cord injury. J Spinal Disord 1996; 9: $367-380$

67c Zhu Q, Ouyang J, Lu W et al. Traumatic instabilities of the cervical spine caused by high-speed axial compression in a human model. An in vitro biomechanical study. Spine 1999; 24: 440-444

\section{Extremitätenverletzungen}

1d Abarbanell NR. Prehospital midthigh trauma and traction splint use: recommendations for treatment protocols. Am J Emerg Med 2001; 19: 137-140

2d Anonymous. Limb trauma. In: School WM, ed. Clinical Practice Guidelines. For use in U.K. Ambulance Services. Guidelines of the Joint Royal Colleges Ambulance Liaison Committee and The Ambulance Service Association. London: Warwick Medical School; 2006. Im Internet: www2.warwick.ac.uk/fac/med/ research/hsri/emergencycare/guidelines/limb_trauma_2006. pdf [Evidenzbasierte Leitlinie]

3d Beck A. Notärztliche Versorgung des Traumapatienten. Notfallund Rettungsmed 2002; 1: 57-61

4d Beck A. Wunde - Fraktur - Luxation. Notfall- und Rettungsmed 2002: 8: 613-624 5d Beck A, Gebhard F, Kinzl L et al. [Principles and techniques of primary trauma surgery management at the site]. Unfallchirurg 2001; 104: 1082 -1096; quiz 1097, 1099

6d Beekley AC, Sebesta JA, Blackbourne LH et al. Prehospital tourniquet use in Operation Iraqi Freedom: effect on hemorrhage control and outcomes. J Trauma 2008; 64: S28 -37; discussion S37

7d Bledsoe B, Barnes D. Traction splint. An EMS relic? JEMS 2004; 29: $64-69$

8d Borschneck AG. Traction splint: proper splint design \& application are the keys. JEMS 2004; 29: 70, 72-75

9d Brodie S, Hodgetts T], Ollerton J et al. Tourniquet use in combat trauma: UK military experience. J R Army Med Corps 2007; 153: 310-313

10d Cuske J. The lost art of splinting. How to properly immobilize extremities \& manage pain. JEMS 2008; 33: 50-64, quiz 66

11d DGU. Leitlinie Polytrauma. 2007. Im Internet: www.dgu-online. de/de/leitlinien/polytrauma.jsp

12d Doyle GS, Taillac PP. Tourniquets: a review of current use with proposals for expanded prehospital use. Prehosp Emerg Care 2008; 12: $241-256$

13d Ficke JR, Pollak AN. Extremity war injuries: Development of clinical treatment principles. J Am Acad Orthop Surg 2007; 15: 590-595

14d Hinds JD, Allen G, Morris CG. Trauma and motorcyclists: born to be wild, bound to be injured? Injury 2007; 38: 1131-1138

15d Irving GA, Noakes TD. The protective role of local hypothermia in tourniquet-induced ischaemia of muscle. J Bone Joint Surg Br 1985; 67: 297-301

16d Kalish J, Burke P, Feldman J et al. The return of tourniquets. Original research evaluates the effectiveness of prehospital tourniquets for civilian penetrating extremity injuries. JEMS 2008; 33: 44-46, 49-50, 52, 54

17d Kragh JF Jr., Walters TJ, Baer DG et al. Practical use of emergency tourniquets to stop bleeding in major limb trauma. J Trauma 2008; 64: S38-49, discussion S49-50

18d Kragh jr. JF, Walters TJ, Baer DG et al. Survival with emergency tourniquet use to stop bleeding in major limb trauma. Ann Surg 2009; 249: 1-7

19d Lackner CK, Lewan U, Deiler S et al. Präklinische Akutversorgung von Amputationsverletzungen. Notfall- und Rettungsmedizin 1999; 2: 188-192

20d Lakstein D, Blumenfeld A, Sokolov T et al. Tourniquets for hemorrhage control on the battlefield: a 4-year accumulated experience. J Trauma 2003; 54: S221-225

21d Lee C, Porter KM. Prehospital management of lower limb fractures. Emerg Med J 2005; 22: 660-663 [LoE 4]

22d Lee C, Porter KM, Hodgetts TJ. Tourniquet use in the civilian prehospital setting. Emerg Med J 2007; 24: 584-587

23d Melamed E, Blumenfeld A, Kalmovich B et al. Prehospital care of orthopedic injuries. Prehosp Disaster Med 2007; 22: 22 -25

24d Perkins T]. Fracture management. Effective prehospital splinting techniques. Emerg Med Serv 2007; 36: 35-37, 39

25d Probst $C$, Hildebrand F, Frink $M$ et al. [Prehospital treatment of severely injured patients in the field: an update]. Chirurg 2007; 78: 875-884 [LoE 5]

26d Quinn RH, Macias DJ. The management of open fractures. Wilderness Environ Med 2006; 17: 41-48 
27d Regel G, Bayeff-Filloff M. [Diagnosis and immediate therapeutic management of limb injuries. A systematic review of the literature]. Unfallchirurg 2004; 107: 919-926 [LoE 3a]

28d Richey SL. Tourniquets for the control of traumatic hemorrhage: a review of the literature. World J Emerg Surg 2007; 2: 28

29d Sackett DL, Richardson WS, Rosenberg W et al. Evidence-based medicine: How to practice and teach EBM. Churchill Livingstone, London 1997

30d Scheinberg S. Traction splint: questioning commended. JEMS 2004; $29: 78$ 31d Slishman S. Traction splint: sins of commission vs. sins of omission. JEMS 2004; 29: 77-78

32d Strohm PC, Bannasch $\mathrm{H}$, Goos M et al. [Prehospital care of surgical emergencies]. MMW Fortschr Med 2006; 148: 34, 36-38

33d Wood SP, Vrahas M, Wedel SK. Femur fracture immobilization with traction splints in multisystem trauma patients. Prehosp Emerg Care 2003; 7: 241 -243

34d Worsing RA Jr. Principles of prehospital care of musculoskeletal injuries. Emerg Med Clin North Am 1984; 2: 205-217 


\section{CME-Fragen}

\section{CME.thieme.de}

\section{CME-Teilnahme}

- Viel Erfolg bei Ihrer CME-Teilnahme unter http://cme.thieme.de

- Diese Fortbildungseinheit ist 12 Monate online für eine

CME-Teilnahme verfügbar.

- Sollten Sie Fragen zur Online-Teilnahme haben, unter http://cme.thieme.de/hilfe finden Sie eine ausführliche Anleitung.

\section{1}

Welche der folgenden Aussagen zur Untersuchung des Thorax in der präklinischen Versorgung trifft zu?

2

2
Welche der folgenden Aussagen
ist falsch?

2
Welche der folgenden Aussagen
ist falsch?
A Eine klinische Untersuchung des Thorax und der Atemfunktion kann durchgeführt werden (GoR 0).

B Die Auskultation der Lunge kann aufgrund häufiger falsch negativer Befunde (Nebengeräusche) unterlassen werden.

C Die Inspektion, Palpation und Perkussion des Thorax können auch in der präklinischen Untersuchung hilfreich sein.

D Die Pulsoxymetrie sollte nur bei beatmeten Patienten angewendet werden.

E In der präklinischen Versorgungsphase sollte eine einmalige Bestimmung der Atemfrequenz und Auskultation der Lunge durchgeführt werden.
Welche der folgenden Aussagen ist falsch?
A Durch die adäquate klinische Untersuchung kann in der Regel ein größerer Pneumothorax in der präklinischen Versorgungsphase weitestgehend ausgeschlossen werden.

B Eine mögliche Progredienz eines kleinen, zunächst klinisch nicht diagnostizierbaren Pneumothorax sollte durch wiederholte Untersuchungen ausgeschlossen werden.

C Bei einer Kombination aus thorakalen Schmerzen, Dyspnoe und fehlendem Atemgeräusch kann bei wachen Patienten ein Pneumothorax mit einer Wahrscheinlichkeit von über $99 \%$ nachgewiesen werden.

D Bei einseitig fehlendem Atemgeräusch muss immer auch die korrekte Tubuslage überprüft werden.

E Aufgrund der schwierigen Verhältnisse in der präklinischen Versorgungsphase handelt es sich der Empfehlung zur Diagnostik eines Pneumothorax um eine Grad-B-Empfehlung.

A Die Verdachtsdiagnose Spannungspneumothorax kann in der präklinischen Untersuchung gestellt werden.

B Die Verdachtsdiagnose Spannungspneumothorax kann bei Nichtvorliegen einer oberen Einflussstauung in Kombination mit einer arteriellen Hypotension ausgeschlossen werden.

C Ein klinisch vermuteter Spannungspneumothorax soll umgehend dekomprimiert werden.

D Bei mit Überdruck beatmeten Patienten kann sich bereits in der präklinischen Versorgungsphase aus einem Pneumothorax ein Spannungspneumothorax entwickeln.

E Ein durch Auskultationsbefund diagnostizierter Pneumothorax kann bei nicht beatmeten Patienten ohne respiratorische Insuffizienz auch unter engmaschiger klinischer Kontrolle beobachtet werden. 


\section{CME-Fragen}

S3-Leitlinie Polytrauma/Schwerverletztenbehandlung

Thoraxtrauma, Schädel-Hirn-Trauma, Wirbelsäulenverletzungen und Extremitätenverletzungen

4

Welche der folgenden Antworten ist falsch?
A Die initiale Entlastung eines Spannungspneumothorax darf nicht durch eine Nadeldekompression erfolgen.

B Die Entlastung eines Spannungspneumothorax kann entweder durch eine Minithorakotomie oder durch die Einlage einer dicken Thoraxdrainage erfolgen.

C Vor Einlage der Thoraxdrainage in den Pleuraraum sollte der Trokar entfernt werden.

D Heimlich-Ventile neigen zur Verstopfung bzw. Funktionsunfähigkeit.

E Bei spontan atmenden Patienten sollten idealerweise geschlossene Beutel- oder Kammersysteme verwendet werden.

\section{5}

Welche der folgenden Antworten zum Schädel-Hirn-Trauma ist falsch?
A Bei Erwachsenen mit Schädel-Hirn-Trauma sollte eine arterielle Normotension mit einem systolischen Blutdruck nicht unter $90 \mathrm{mmHg}$ angestrebt werden.

B Ein Absinken der arteriellen Sauerstoffsättigung unter $90 \%$ sollte vermieden werden.

C Die wiederholte Erfassung und Dokumentation von Bewusstseinsklarheit, Bewusstseinstrübung oder Bewusstlosigkeit mit Pupillenfunktion und Glasgow Coma Scale soll erfolgen.

D Beim Schädel-Hirn-Trauma soll die Gabe von Glukokortikoiden zur Hirnödemprophylaxe erwogen werden.

E Perforierende Gegenstände sollten auch bei Schädel-Hirn-Trauma-Verletzungen belassen und erst in der Klinik entfernt werden.
6

Welche der folgenden Aussagenkombinationen ist richtig? Bei Verdacht auf stark erhöhten intrakraniellen Druck, insbesondere Zeichen der transtentoriellen Herniation, können folgende Maßnahmen angewendet werden:
1. Hyperventilation
2. Mannitolgabe
3. Hypertone Kochsalzlösung
4. Kortikoidgabe
5. Oberkörperhochlagerung bei normalem systolischem Blutdruck
A Nur die Aussagen 1, 2, 3 und 5 sind richtig.
B Nur die Aussagen 1 und 3 sind richtig.
C Alle Aussagen sind richtig.
D Nur die Aussagen 1, 2 und 4 sind richtig.
E Nur die Aussagen 1, 3, 4 und 5 sind richtig.

\section{7}

Welche der folgenden Aussagen zu Verletzungen der Wirbelsäule trifft nicht zu?
A Auch in der Präklinik soll eine gezielte körperliche Untersuchung inklusive der Wirbelsäule und der mit ihr verbundenen Funktionen durchgeführt werden.

B Bei bewusstlosen Patienten soll bis zum Beweis des Gegenteils von dem Vorliegen einer Wirbelsäulenverletzung ausgegangen werden.

C Eine instabile Wirbelsäulenverletzung kann in der präklinischen Versorgung nicht ausgeschlossen werden.

D Akutschmerzen im Wirbelsäulenbereich nach Trauma sollten als ein Hinweis auf eine Wirbelsäulenverletzung gewertet werden.

E Bei inkompletter Querschnittssymptomatik aufgrund einer Wirbelsäulenverletzung kann die Glukokortikoidgabe erwogen werden. 


\section{CME-Fragen}

S3-Leitlinie Polytrauma/Schwerverletztenbehandlung

Thoraxtrauma, Schädel-Hirn-Trauma, Wirbelsäulenverletzungen und Extremitätenverletzungen

8

Welche der folgenden Aussagen zur Verletzung einer Wirbelsäule trifft zu?
A Bei akuter Lebensbedrohung (zum Beispiel Feuer oder Explosionsgefahr) ist die sofortige Rettung aus dem Gefahrenbereich nur unter Berücksichtigung aller notwendigen Stabilisierungsmaßnahmen an der Wirbelsäule durchzuführen.

B Die Halswirbelsäule soll vor der eigentlichen technischen Rettung immobilisiert werden.

C Patienten mit neurologischen Ausfällen und vermuteter Wirbelsäulenverletzung sollten primär nur in überregionale Traumazentren mit Wirbelsäulenchirurgie transportiert werden.

D Der Transport von Patienten mit instabiler Wirbelsäulenverletzung muss vor allem schnell und ggf. auch ohne adäquate Ruhigstellung der Wirbelsäule erfolgen.

E Intubation von Patienten mit HWS-Läsion darf nur unter vorheriger Anlage eines Stiff-Neck erfolgen.

\section{9}

Welche Antwort zu Verletzungen der Extremitäten trifft nicht zu?
A Stark blutende Verletzungen der Extremitäten, welche die Vitalfunktion beeinträchtigen können, sollten mit Priorität versorgt werden.

B Die Versorgung von Verletzungen der Extremitäten soll weitere Schäden vermeiden und die Gesamtrettungszeit bei Vorliegen weiterer Verletzungen nicht verzögern.

C Alle Extremitäten eines Verunfallten sollten präklinisch orientierend untersucht werden.

D Eine nur vermutlich verletzte Extremität braucht vor dem Transport des Patienten nicht unbedingt ruhiggestellt werden.

E Grob dislozierte Frakturen und Luxationen sollten wenn möglich und insbesondere bei begleitender Ischämie der betroffenen Extremität präklinisch annähernd reponiert werden.

\section{0}

Welche der Aussagen sind richtig? Zum Stufenschema bei aktiven Blutungen gehören folgende Maßnahmen:
1. Manuelle Kompression

2. Druckverband

3. Hochlagerung

4. Tourniquet-Behandlung

5. Primäres direktes Abklemmen der Gefäße

A Nur die Aussagen 1, 2, 3 und 5 sind richtig.

B Nur die Aussagen 1 und 2 sind richtig.

C Alle Aussagen sind richtig.

D Nur die Aussagen 1, 2, 3 und 4 sind richtig.

E Nur die Aussagen 1,2 und 3 sind richtig. 